\title{
Hypersonic Flow Field Reconfiguration and Drag Reduction of Blunt Body with Spikes and Sideward Jets
}

\author{
Guilai Han (D) and Zonglin Jiang \\ State Key Laboratory of High Temperature Gas Dynamics, Institute of Mechanics, Chinese Academy of Sciences, Beijing, China \\ Correspondence should be addressed to Guilai Han; hanguilai@imech.ac.cn
}

Received 13 April 2017; Revised 3 January 2018; Accepted 12 February 2018; Published 24 April 2018

Academic Editor: Paul Williams

Copyright ( 2018 Guilai Han and Zonglin Jiang. This is an open access article distributed under the Creative Commons Attribution License, which permits unrestricted use, distribution, and reproduction in any medium, provided the original work is properly cited.

\begin{abstract}
Flow field reconfiguration and drag reduction have been investigated by numerically solving Navier-Stokes equations, with the 2nd order dispersion controlled dissipative scheme adopted for the convective term discretion and the 2nd order central difference scheme for viscous term. Spikes and sideward jets nearby the spike noses were set up in the front of the blunt body to reconfigure the flow field and reduce drag during hypersonic flight. The spikes and the sideward jets led to the formation of the conical shock, the reattached shock, and the circumfluence, instead of a bow shock. According to the quantitative analysis, the reconfiguration of the flow field played a dominant role in drag reduction. Basing on the locations of the reattached shock and its interaction with the conical shock, a combined parameter was proposed for a primary criterion to design the spiked bodies with sideward jets for the purpose of drag reduction.
\end{abstract}

\section{Introduction}

The concept of a spiked blunt body was first proposed by Bogdonoff [1]. However, investigations of flow separation in front of blunt bodies at supersonic speeds have been made since the early 1950s [2-5]. Research has shown that spikes can construct a separation zone over blunt bodies to significantly lower the aerodynamic heat rate and pressure distribution, which was valuable for thermal protection and drag reduction. Such a simple and efficient configuration has made it appealing. In the 1960s, much research was conducted on separation characteristics and corresponding flow instability. Maull investigated unsteady flow field properties caused by spike length and shape on blunt bodies, concluding that flow oscillation was due to two factors, separation induced by shock wave and flow reattachment [6]. Wood investigated spiked cone cylinders flying at Mach 10 and defined five different flow patterns and corresponding scopes using a combination of spike lengths and cone angles [7]. Hahn introduced mass injection at different spiked blunt body positions flying at Mach 3.3, including the tip, base, and the reattachment region [8]. Reding et al. considered unsteady aerodynamics for a spiked drag reduction device based on structure deflection coupled with thermal expansion caused by aerodynamic heating [9]. Calarese and Hankey experimentally and numerically studied shockwave oscillation modes on a spiked body at Mach 3, observing both symmetrical and asymmetrical oscillations that demonstrated and confirmed the existence of standing rotational waves and their corresponding effects [10]. A study by Hutt and Howe first considered the preliminary optimization design of vehicle configuration, in which the effects of spikes on bodies with different cross sections were compared by measuring force coefficients and the pressure center in a supersonic wind tunnel [11].

Alongside the rapid expansion of computer technology beginning in the 1990s, many studies on numerical simulations and solutions have allowed for a remarkable level of understanding with regards to spiked blunt bodies. Cooperation between experiments and numerical simulationss validated many of these studies. Mehta studied the aerodynamic heat flux and pressure distribution over spiked bodies at Mach 6.8 and the relationship between them [12]. Milićev et al. studied the influence of spike shape on aerodynamic coefficients at supersonic flow past blunt-nosed bodies, including drag, lift, pitching moment, and pressure center 
[13]. Panaras and Drikakis investigated spiked blunt bodies in supersonic and hypersonic flow and showed that unsteady flow was characterized by periodic radial inflation and conical separation bubble collapse [14]. They also pointed out that there was a certain spike length, beyond which flow is stable. Menezes et al. studied spiked blunt body drag in a hypersonic shock tunnel and showed that drag reduction significantly decreased as attack angle increased. In some critical cases, the spiked blunt body changed into a resistance element $[15,16]$. Morgenstern showed that an asymmetric flow field in a three-dimensional simulation resulted in unsteady fluctuation during flight with attack angle, leading to axial pulsation and normal forces, which resulted in an extreme disadvantage for flight and controlling [17]. Thurman [18] and Schulein [19] achieved some positive results by changing aerodynamic characteristics utilizing a rotating spike, which required a special system to control and rotate the rod and was too complicated for practical application. Liu and Jiang gave some experimental investigations on pressure distribution and flow field structure of the spiked blunt body with unexpanded sideward jets [20]. Ma et al. furtherly investigated the flow unsteadiness in the circumfluence formed by the spiked bodies [21]. Eghlima and Mansour developed a method for drag reduction by the combination of a spike and a counter jet on blunt bodies [22]. Bibi et al. numerically investigated the use of the active opposing jet concept in combination with geometric variations of the opposing jet nozzle to alleviate high wave drag formation [23]. Huang et al. numerically studied the influence of the cavity configuration on the drag and heat flux reduction mechanism of a blunt body [24]. Han set up the new nonablation concept utilizing adaptive drag reduction and thermal protection system (NADTPS) for hypersonic vehicles basing on a spiked blunt body with sideward jet nearby the spike nose [25]. The NADTPS uses aerodynamic heating on the blunt body and pressure changes coolant into vapor, thus generating a sideward jet and convective cooling at the spike nose, preventing the spike from being destroyed. This sideward jet can lower shock wave interaction on the blunt body shoulder, thus optimizing aerodynamic characteristics and efficiently demonstrating experiments in a hypersonic tunnel with an attack angle.

This paper investigated the spiked blunt bodies with and without sideward jets flying at hypersonic speed, using numerical simulations. The incoming flow Mach number changed from 5 to 10 , and the spike length ratio over the blunt body diameter changed from 0.5 to 1.0. The flow structure reconfiguration, especially the shock wave structure, was analyzed by flow field visualization. Aerodynamic force characteristics were calculated and compared with those of blunt bodies to analyze drag reduction effects and the corresponding mechanisms.

\section{Governing Equations and Numerical Methods}

The two-dimensional axisymmetric compressible NavierStokes equation set was applied as the governing equations, with the thermally perfect gas model and ideal gas state equation [26]. The flow medium can be considered as a single component with diffusion neglected. Under the Jacobian transformation between physical space and computational space, the governing equation is

$$
\frac{\partial \tilde{\mathbf{U}}}{\partial t}+\frac{\partial \tilde{\mathbf{E}}}{\partial \xi}+\frac{\partial \tilde{\mathbf{F}}}{\partial \eta}+\frac{1}{y} \tilde{\mathbf{H}}=\frac{1}{\operatorname{Re}}\left(\frac{\partial \tilde{\mathbf{E}}_{v}}{\partial \xi}+\frac{\partial \tilde{\mathbf{F}}_{v}}{\partial \eta}+\frac{1}{y} \tilde{\mathbf{H}}_{v}\right)
$$

and each vector component transformation can be written as follows:

$$
\begin{aligned}
\tilde{\mathbf{U}} & =\frac{1}{J} \mathbf{U}, \\
\tilde{\mathbf{E}} & =\frac{1}{J}\left(\xi_{x} \mathbf{E}+\xi_{y} \mathbf{F}\right), \\
\tilde{\mathbf{F}} & =\frac{1}{J}\left(\eta_{x} \mathbf{E}+\eta_{y} \mathbf{F}\right), \\
\tilde{\mathbf{H}} & =\frac{1}{J} \mathbf{H}, \\
\tilde{\mathbf{E}}_{v} & =\frac{1}{J}\left(\xi_{x} \mathbf{E}_{v}+\xi_{y} \mathbf{F}_{v}\right), \\
\tilde{\mathbf{F}}_{v} & =\frac{1}{J}\left(\eta_{x} \mathbf{E}_{v}+\eta_{y} \mathbf{F}_{v}\right), \\
\tilde{\mathbf{H}}_{v} & =\frac{1}{J} \mathbf{H}_{v},
\end{aligned}
$$

where $\xi$ and $\eta$ stand for computational space, and $x$ and $y$ stand for physical space. $J$ stands for the Jacobian determinant:

$$
J=\left|\frac{\partial(\xi, \eta)}{\partial(x, y)}\right|=\frac{1}{x_{\xi} y_{\eta}-x_{\eta} y_{\xi}}
$$

The detailed formula for each vector in (2) can be written as follows:

$$
\begin{gathered}
\mathbf{U}=\left[\begin{array}{c}
\rho \\
\rho u \\
\rho v \\
E
\end{array}\right], \\
\mathbf{E}=\left[\begin{array}{c}
\rho u \\
\rho u^{2}+p \\
\rho u v \\
(E+p) u
\end{array}\right], \\
\mathbf{F}=\left[\begin{array}{c}
\rho v \\
\rho u v \\
\rho v^{2}+p \\
(E+p) v
\end{array}\right],
\end{gathered}
$$




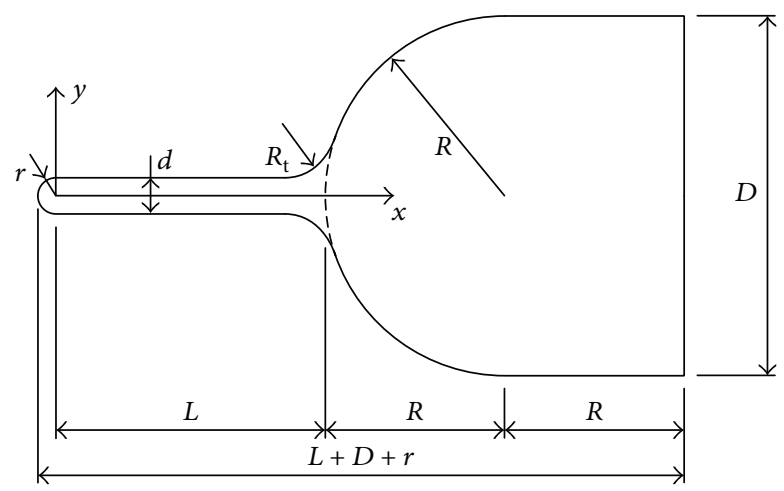

(a)

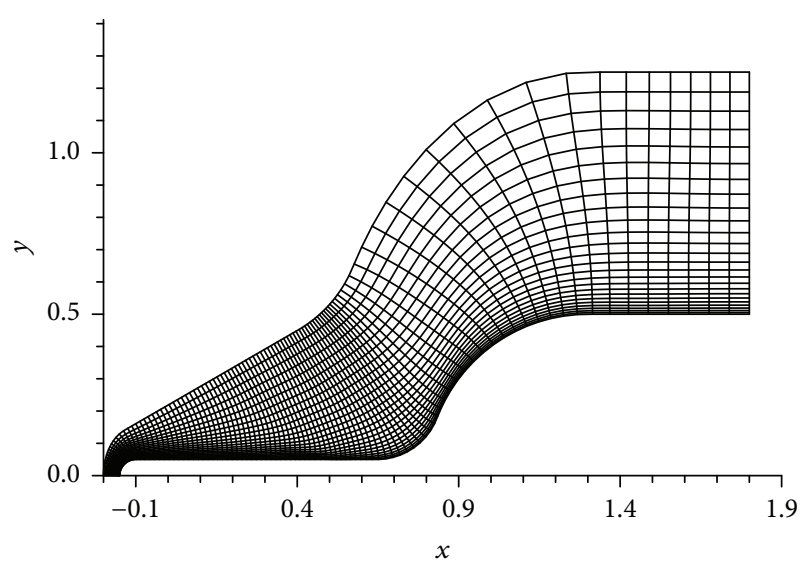

(b)

FIGURE 1: (a) Configuration of the spiked blunt body for simulation. (b) Grid system for the spiked blunt body with spike length of $L / D=0.9$.

$$
\begin{aligned}
& \mathbf{H}=\left[\begin{array}{c}
\rho v \\
\rho u v \\
\rho v^{2} \\
(E+p) v
\end{array}\right], \\
& \mathbf{E}_{v}=\left[\begin{array}{c}
0 \\
\tau_{x x} \\
\tau_{x y} \\
u \tau_{x x}+v \tau_{x y}-q_{x}
\end{array}\right] \text {, } \\
& \mathbf{F}_{v}=\left[\begin{array}{c}
0 \\
\tau_{x y} \\
\tau_{y y} \\
u \tau_{x y}+v \tau_{y y}-q_{y}
\end{array}\right] \text {, } \\
& \mathbf{H}_{v}=\left[\begin{array}{c}
0 \\
\tau_{x y}-\frac{2}{3} y \frac{\partial}{\partial x}\left(\frac{\mu v}{y}\right) \\
\tau_{y y}-\tau_{\theta \theta}-\frac{2}{3} \frac{\mu v}{y}-\frac{2}{3} y \frac{\partial}{\partial y}\left(\frac{\mu v}{y}\right) \\
u \tau_{x y}+v \tau_{y y}-\frac{2}{3} \frac{\mu v^{2}}{y}-\frac{2}{3} y \frac{\partial}{\partial y}\left(\frac{\mu v^{2}}{y}\right)-\frac{2}{3} y \frac{\partial}{\partial x}\left(\frac{\mu u v}{y}\right)-q_{y}
\end{array}\right], \\
& \tau_{x x}=\mu\left(\frac{4}{3} \frac{\partial u}{\partial x}-\frac{2}{3} \frac{\partial v}{\partial y}\right), \\
& \tau_{y y}=\mu\left(\frac{4}{3} \frac{\partial v}{\partial y}-\frac{2}{3} \frac{\partial u}{\partial x}\right), \\
& \tau_{x y}=\tau_{y x}=\mu\left(\frac{\partial u}{\partial y}+\frac{\partial v}{\partial x}\right), \\
& \tau_{\theta \theta}=\mu\left[-\frac{2}{3}\left(\frac{\partial u}{\partial x}+\frac{\partial v}{\partial y}\right)+\frac{4}{3} \frac{v}{y}\right], \\
& q_{x}=-\frac{\mu}{\operatorname{Pr}(\gamma-1) M_{\infty}^{2}} \frac{\partial T}{\partial x},
\end{aligned}
$$

$$
\begin{aligned}
& q_{y}=-\frac{\mu}{\operatorname{Pr}(\gamma-1) M_{\infty}^{2}} \frac{\partial T}{\partial y}, \\
& q_{z}=-\frac{\mu}{\operatorname{Pr}(\gamma-1) M_{\infty}^{2}} \frac{\partial T}{\partial z} .
\end{aligned}
$$

The viscosity coefficient $\mu$ was calculated with Sutherland formula, and the heat conduction coefficient $k$ can be calculated via the Prandtl number definition derived as $\operatorname{Pr}=k / \mu c_{\mathrm{p}}$, where $c_{\mathrm{p}}$ denotes the specific heat at constant pressure and $\operatorname{Pr}=0.72$.

Jiang et al. analyzed the dispersion terms in the modified equation and theoretically derived the dispersion conditions for nonoscillatory shock capturing schemes, on which a series of dispersion controlled dissipative (DCD) schemes were set up $[27,28]$. The 2 nd order DCD scheme was adopted to discrete the convective terms, and the StegerWarming method [29] was applied for flux vector splitting. The 2nd order central difference scheme [26] was used for viscous term discretization. The explicit method was used for time integration, with the CFL condition for time-step calculation and scheme stability requirements. The local time step method [30] was introduced to accelerate the simulation convergence process using different time steps at grid points.

The configuration and dimension of the spiked blunt body are shown in Figure 1(a). The diameter $D$ of the spherical head cylinder was chosen as the characteristic length, and the diameter of the spike was set to $d / D=0.1$. The $L / D$ denotes spike length and was set from 0.5 to 1.0 with an interval of $0.1 . R_{\mathrm{t}}$ stands for transition part radius at the spike root and was set as $R_{\mathrm{t}} / D=0.2$. The sideward jet position was set at the range from $(0, r)$ to $(d / 10, r)$ nearby the spike nose in the Cartesian coordinate system. The physical model and computational domain are shown in Figure 1(b). The RL method [31] was applied for grid generation by solving an elliptic partial differential equation set. The spiked blunt body model and the corresponding grid system are shown in Figure 1(b), in which orthogonality is satisfied in each grid system and refined by algebraic method near the wall boundary. 


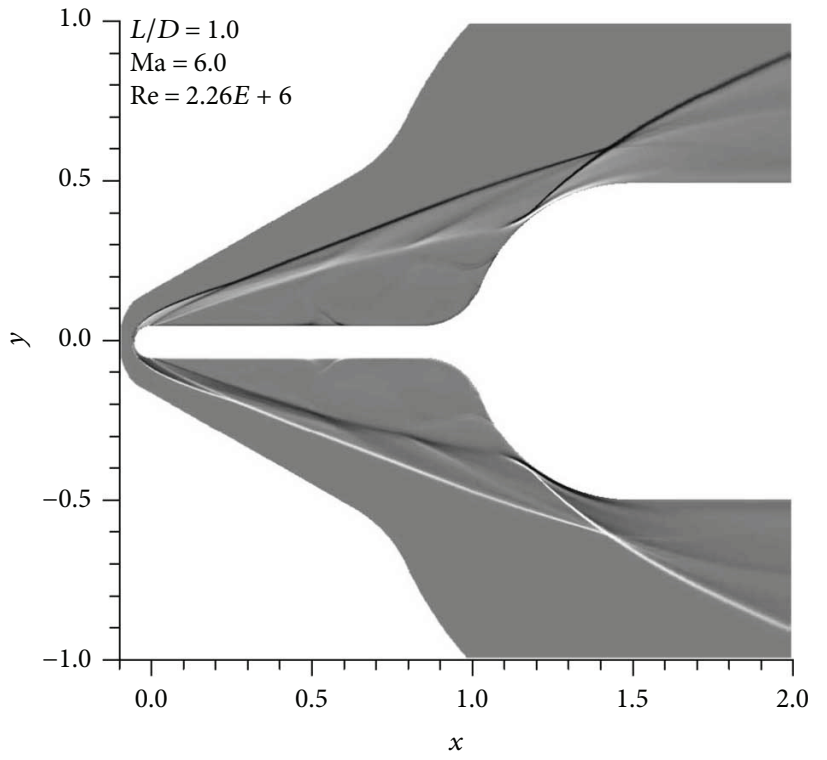

(a)

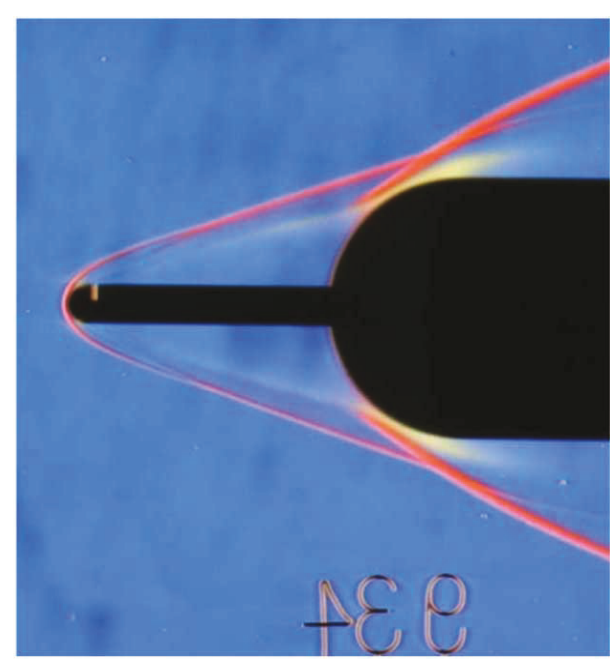

(b)

Figure 2: Flow field wave structure of spiked blunt body with spike length $L / D=1.0$; (a) numerical Schlieren photograph; (b) experimental Schlieren photograph [20].

Four different grid resolutions have been applied for primary investigation, including $101 \times 401,101 \times 801,201 \times 801$, and $401 \times 1601$. Much finer structure can be captured by the resolution of both $201 \times 801$ and $401 \times 1601$, especially the shock waves, spline line, and flow structure interactions. And the results basing on the resolution of $201 \times 801 \mathrm{can}$ match the result of the finer resolution, which can get to a convergent solution much faster. Therefore, the computational grids were set as 201 grid points in a vertical direction to the body surface and 801 in the direction along the surface. The boundary condition was set as a constant temperature wall with $T_{\mathrm{w}}=300.0 \mathrm{~K}$. The sideward jets were set to be sonic jets, where the static temperature and density were equal to the local flow condition, and the speed equal to the corresponding sound speed. And the sideward jet was set as a slit with the width of $d / 10$ near by the spike nose from $(0, r)$ to $(d / 10, r)$ in the Cartesian coordinate system in Figure 1.

The numerical solution validation was carried out by comparing the numerical wave structure and experimental flow field as shown in Figure 2. The conditions were set as $M_{\infty}=6.0$ and $L / D=1.0$. Numerical and experimental results are in agreement, indicating the main flow field structures, including conical shock, reattached shock, slip line, shear layer, and separation zone. Aerodynamic force validation calculations utilized flow simulation around a sphere flying at hypersonic speed with the diameter of $0.1 \mathrm{~m}$ as listed in Table 1. The drag coefficient scope was from 0.8713 to 0.8910 in the simulations, close to the 0.87 to 0.90 scope identified in the experiments [32].

\section{Results and Discussion}

3.1. Typical Flow Field Structure. Former investigations on spiked blunt bodies usually focused on the formation and
TABLE 1: Incoming flow condition and configuration of the spiked body.

\begin{tabular}{lcc}
\hline$M_{\infty}$ & $\operatorname{Re}_{\infty} / 10^{6} \mathrm{~m}^{-1}$ & $L / D$ \\
\hline 5.0 & 1.88 & $0.5-1.0$ \\
6.0 & 2.26 & $0.5-1.0$ \\
7.0 & 2.64 & $0.5-1.0$ \\
8.0 & 3.02 & $0.5-1.0$ \\
9.0 & 3.39 & $0.5-1.0$ \\
10.0 & 3.77 & $0.5-1.0$ \\
\hline
\end{tabular}

stability of the separation zone. However, this paper considers that drag reduction mainly depends on wave drag reduction due to flow field reconfiguration, in which the bow shock is changed into a conical shock interacting with a reattached shock. In this section, the shock wave structures around the spiked blunt bodies are illustrated using 6 different Mach numbers and 6 different spike lengths, including those with spiked blunt bodies and sideward sonic jets near the spike nose, as shown in Figures 3-14.

The typical shock wave structures of pure spiked blunt bodies with defined labels are shown in Figure 15. Density gradient in the $y$ direction was used to describe a Schlieren photograph and reconfigured flow field. The spike nose became the new stagnation with a little bow shock in front and conical shock around. High-speed flow after the conical shock traveled to the blunt body shoulder, leading to reattachment. Hence, the reattached shock was formed on the blunt body shoulder, resulting in a zone with high pressure. The inverse pressure gradient caused some of the gas flow to the spike nose, in the opposite direction of incoming flow. Thus, a circumfluence was generated around the spike and the blunt body nose. In the circumfluence, the gas flow 


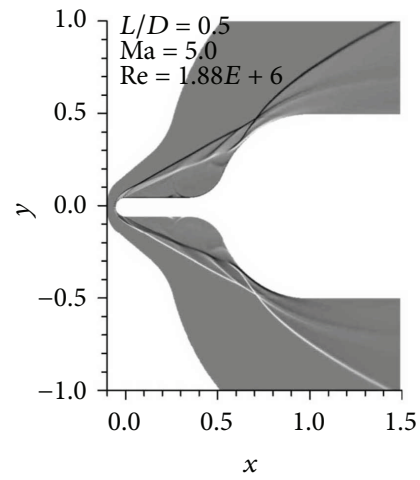

(a) $M_{\infty}=5.0$

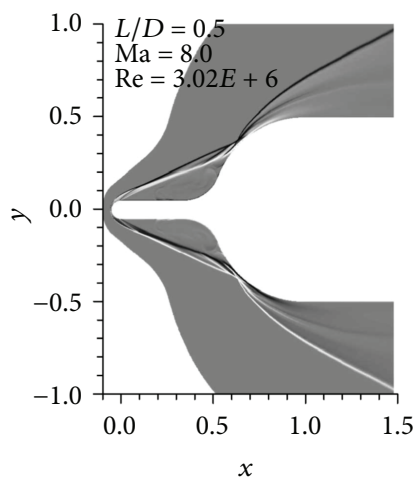

(d) $M_{\infty}=8.0$

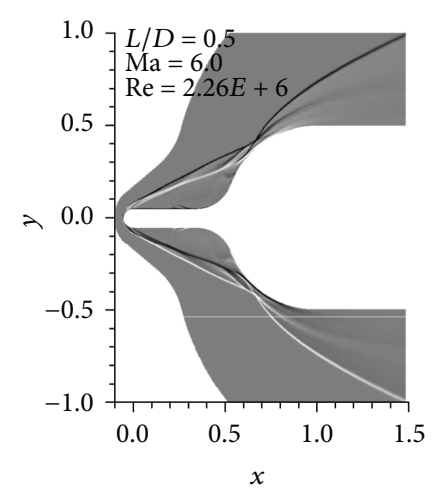

(b) $M_{\infty}=6.0$

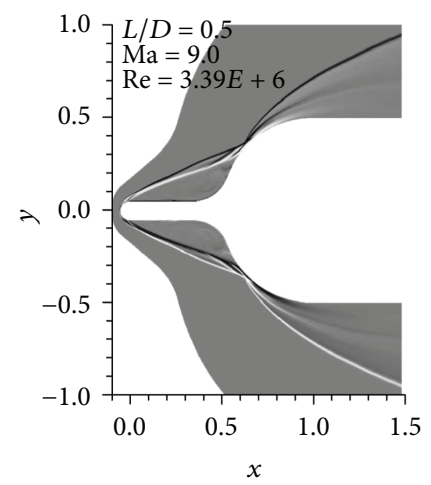

(e) $M_{\infty}=9.0$

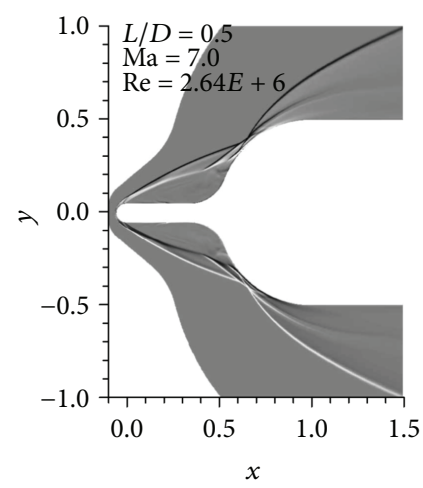

(c) $M_{\infty}=7.0$

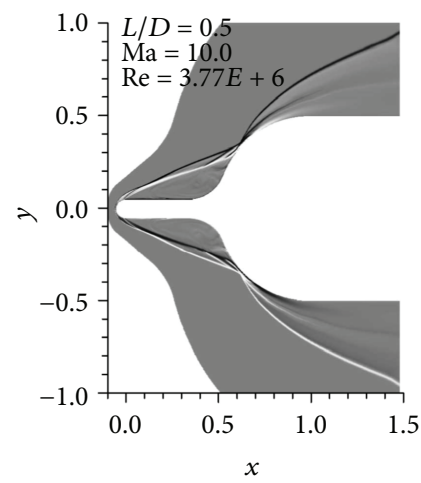

(f) $M_{\infty}=10.0$

FIGURE 3: Flow field density gradient in $y$ direction of pure spiked blunt bodies. Spike length $L / D=0.5$.

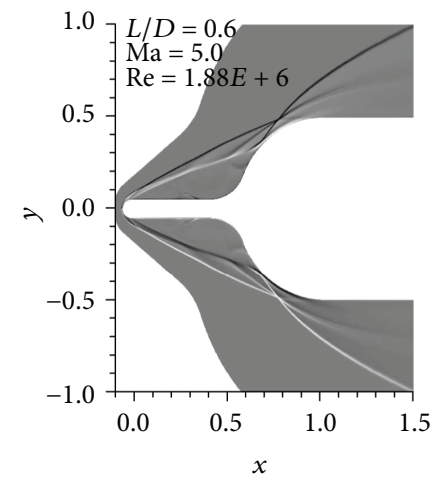

(a) $M_{\infty}=5.0$

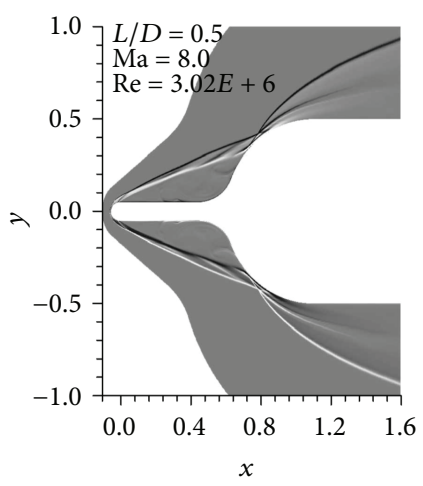

(d) $M_{\infty}=8.0$

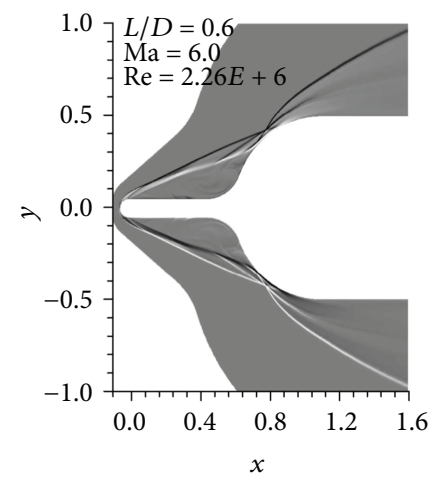

(b) $M_{\infty}=6.0$

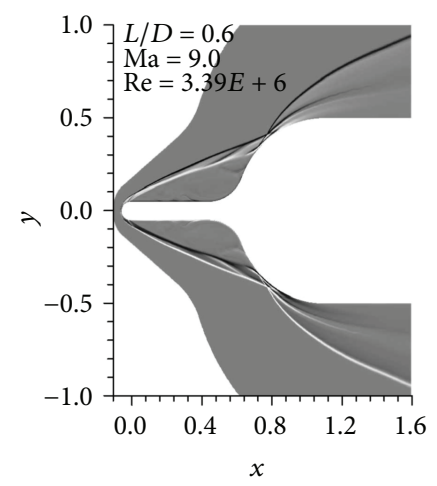

(e) $M_{\infty}=9.0$

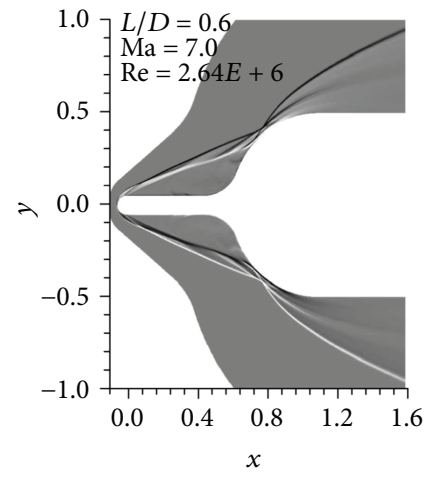

(c) $M_{\infty}=7.0$

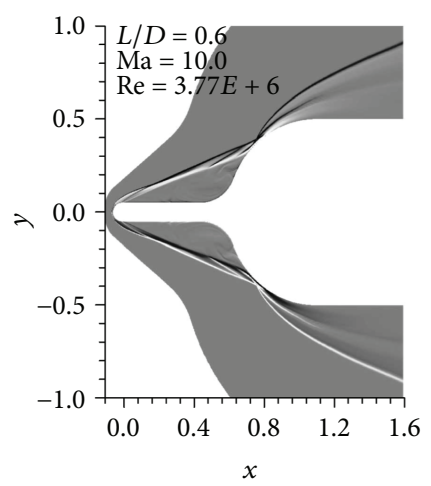

(f) $M_{\infty}=10.0$

FIGURE 4: Flow field density gradient in $y$ direction of pure spiked blunt bodies. Spike length $L / D=0.6$. 


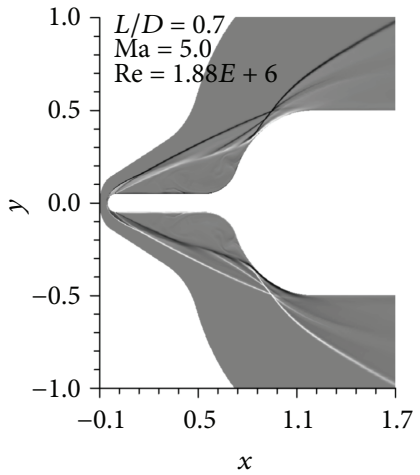

(a) $M_{\infty}=5.0$

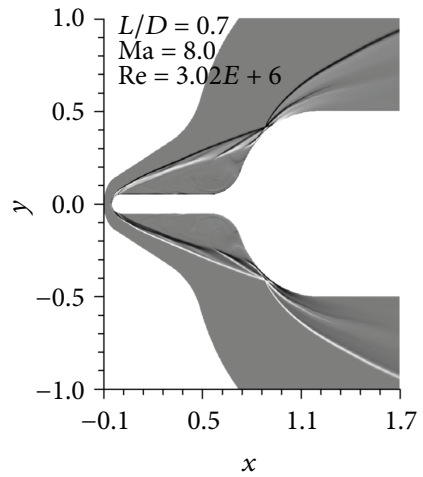

(d) $M_{\infty}=8.0$

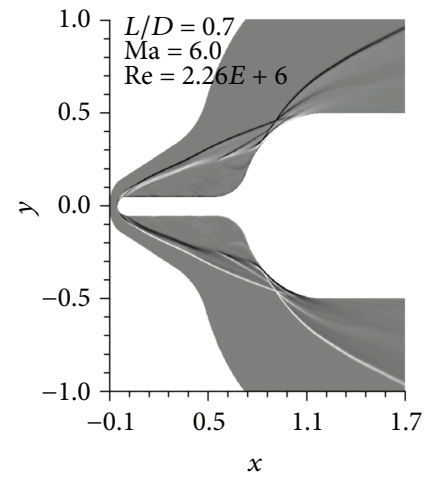

(b) $M_{\infty}=6.0$

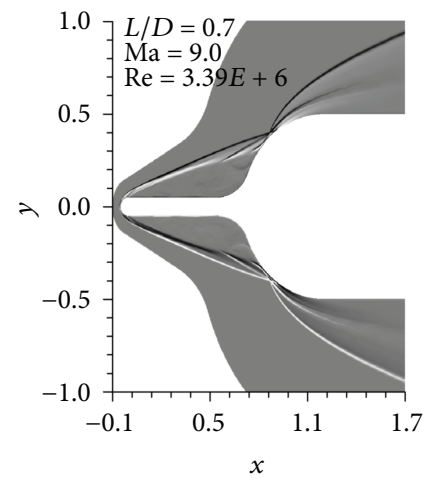

(e) $M_{\infty}=9.0$

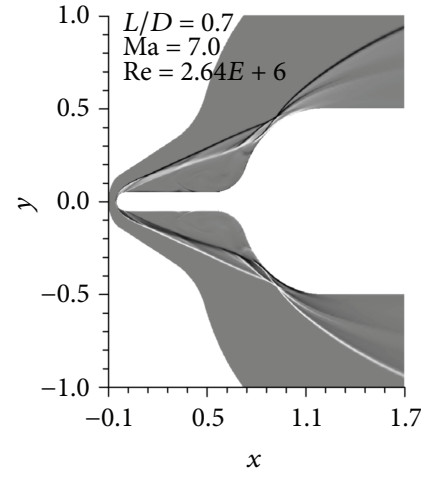

(c) $M_{\infty}=7.0$

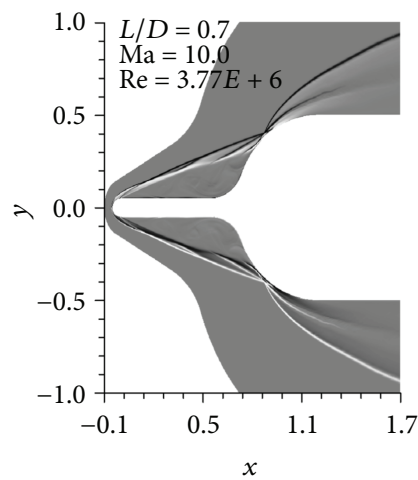

(f) $M_{\infty}=10.0$

FIGURE 5: Flow field density gradient in $y$ direction of pure spiked blunt bodies. Spike length $L / D=0.7$.

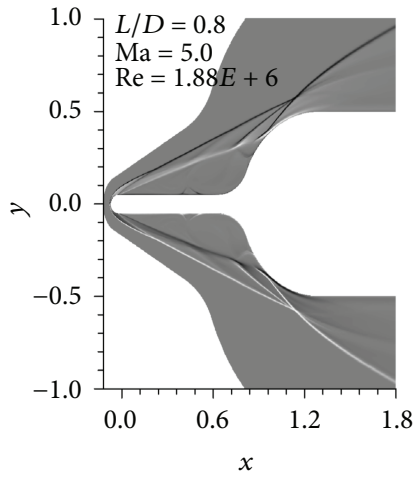

(a) $M_{\infty}=5.0$

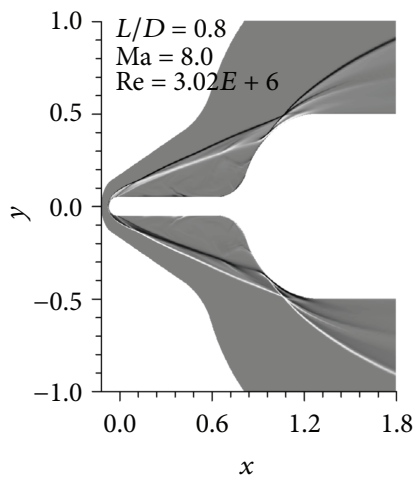

(d) $M_{\infty}=8.0$

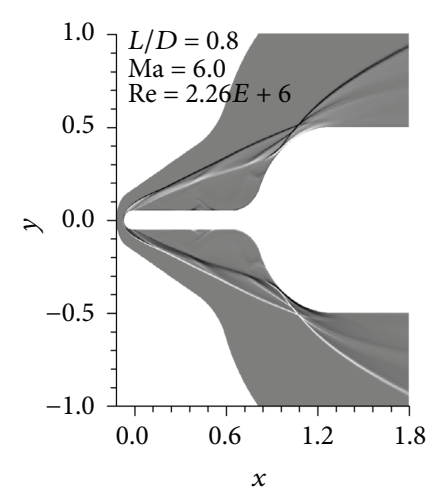

(b) $M_{\infty}=6.0$

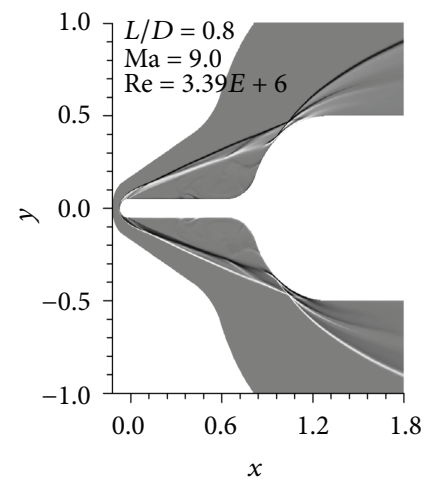

(e) $M_{\infty}=9.0$

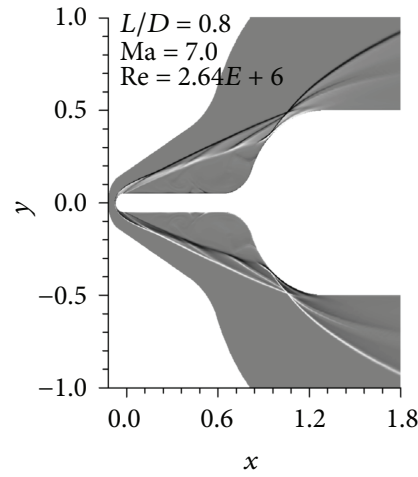

(c) $M_{\mathrm{\infty}}=7.0$

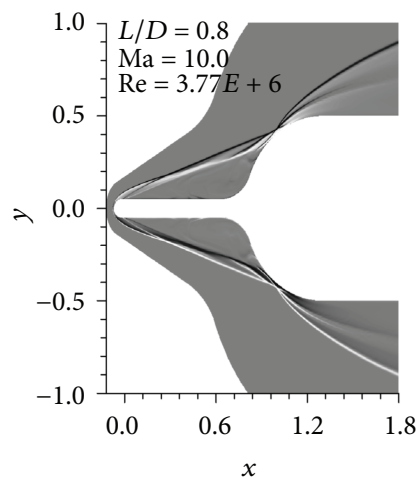

(f) $M_{\infty}=10.0$

FIGURE 6: Flow field density gradient in $y$ direction of pure spiked blunt bodies. Spike length $L / D=0.8$. 


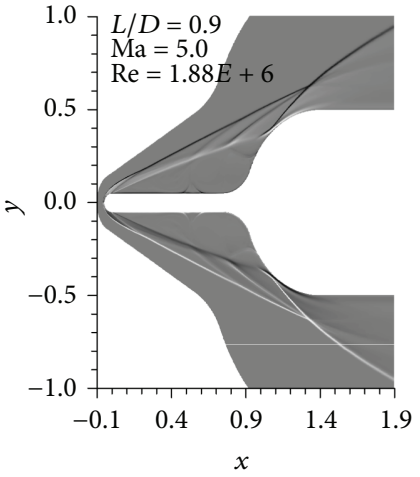

(a) $M_{\infty}=5.0$

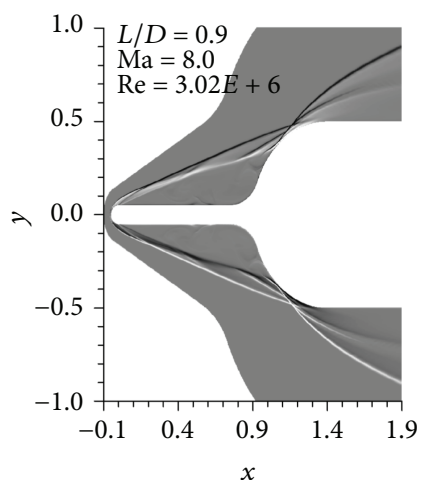

(d) $M_{\infty}=8.0$

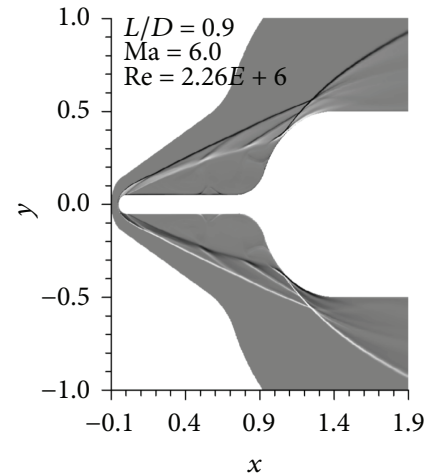

(b) $M_{\infty}=6.0$

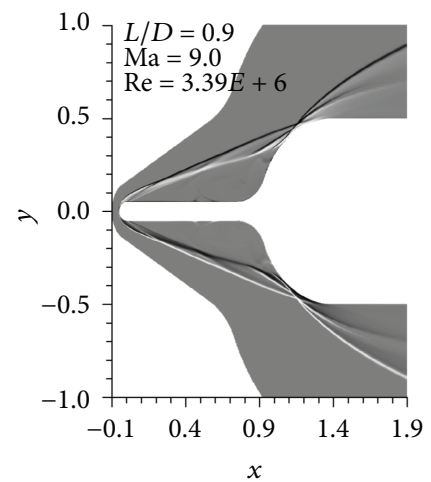

(e) $M_{\infty}=9.0$

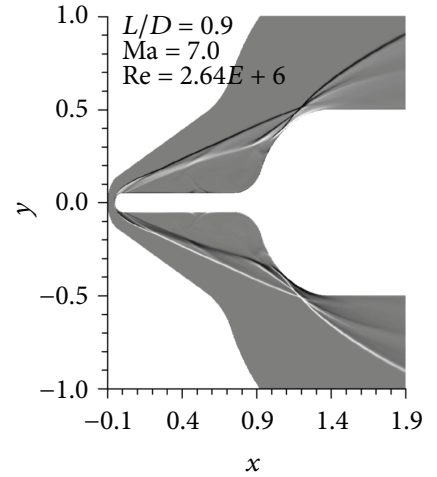

(c) $M_{\infty}=7.0$

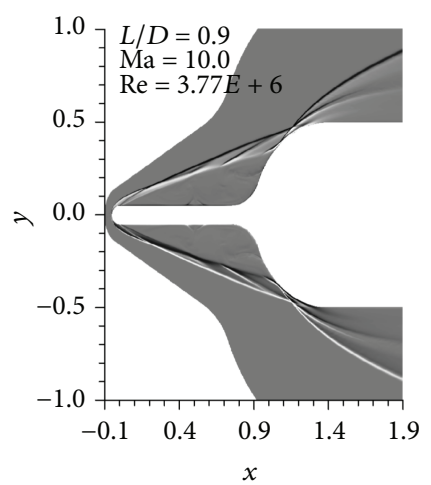

(f) $M_{\infty}=10.0$

Figure 7: Flow field density gradient in $y$ direction of pure spiked blunt bodies. Spike length $L / D=0.9$.

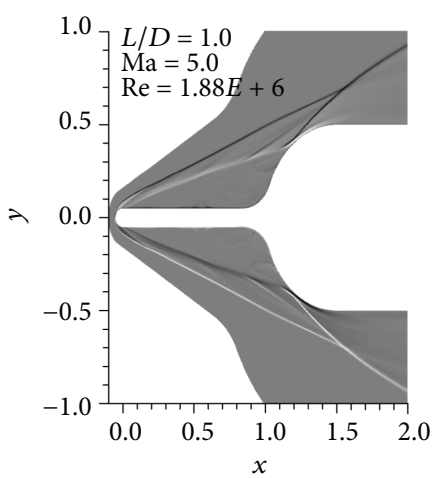

(a) $M_{\infty}=5.0$

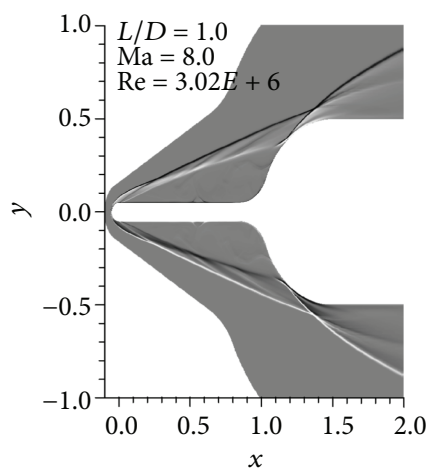

(d) $M_{\infty}=8.0$

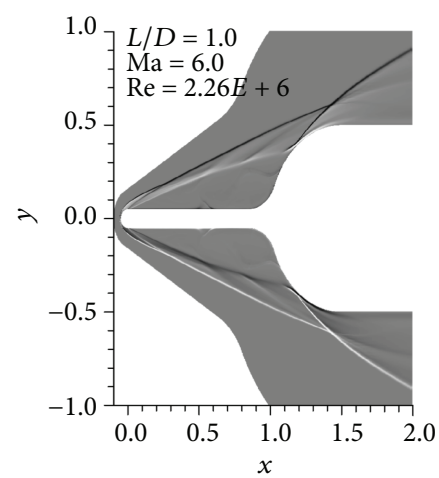

(b) $M_{\infty}=6.0$

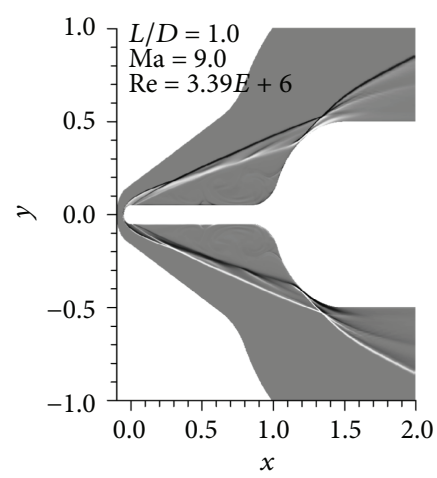

(e) $M_{\infty}=9.0$

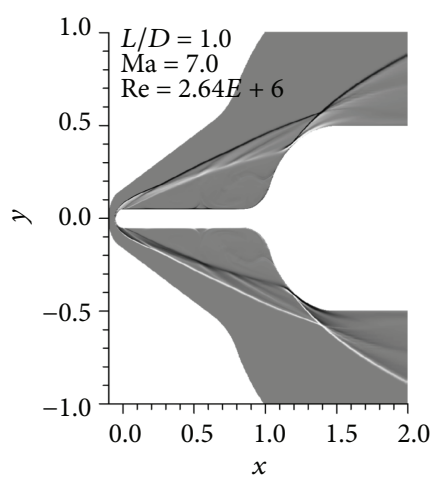

(c) $M_{\infty}=7.0$

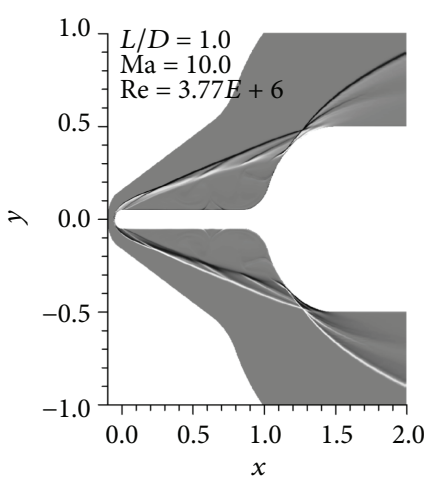

(f) $M_{\infty}=10.0$

FIGURE 8: Flow field density gradient in $y$ direction of pure spiked blunt bodies. Spike length $L / D=1.0$. 


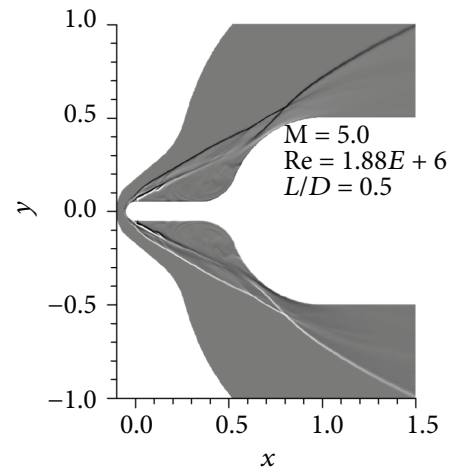

(a) $M_{\infty}=5.0$

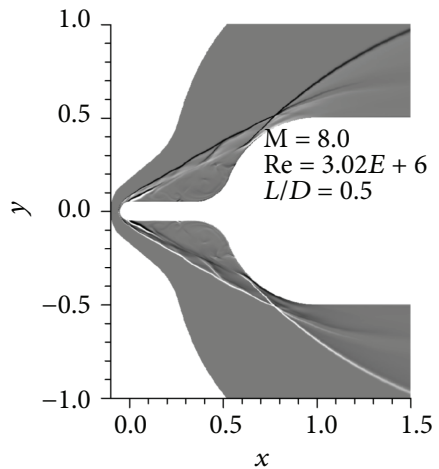

(d) $M_{\infty}=8.0$

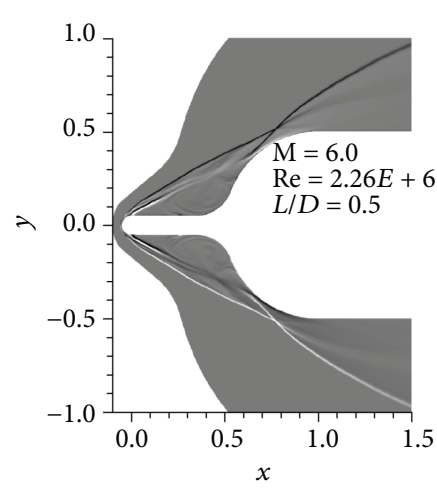

(b) $M_{\infty}=6.0$

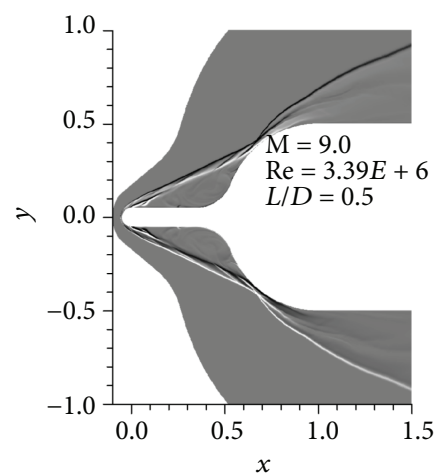

(e) $M_{\infty}=9.0$

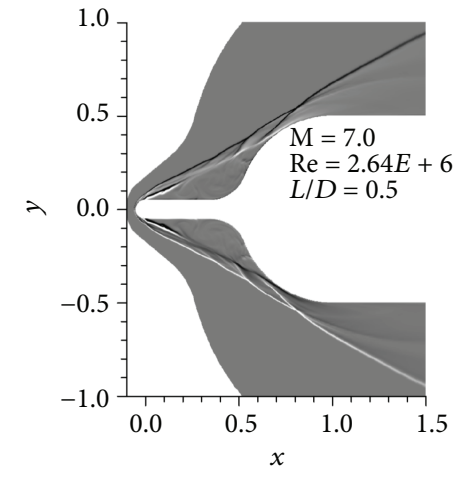

(c) $M_{\infty}=7.0$

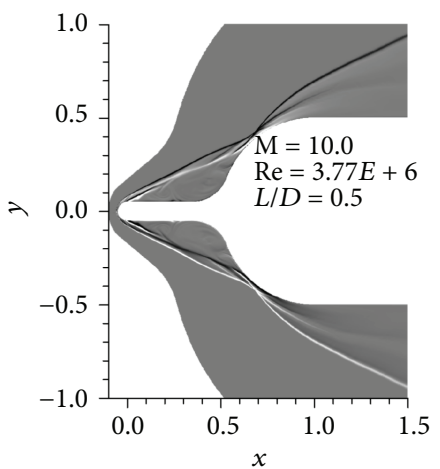

(f) $M_{\infty}=10.0$

Figure 9: Flow field density gradient in $y$ direction of spiked blunt bodies with the sideward sonic jet. Spike length $L / D=0.5$.

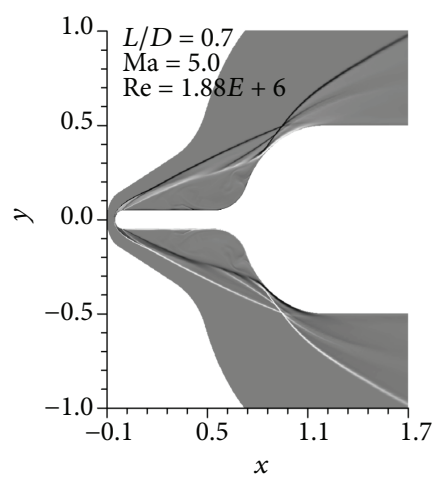

(a) $M_{\infty}=5.0$

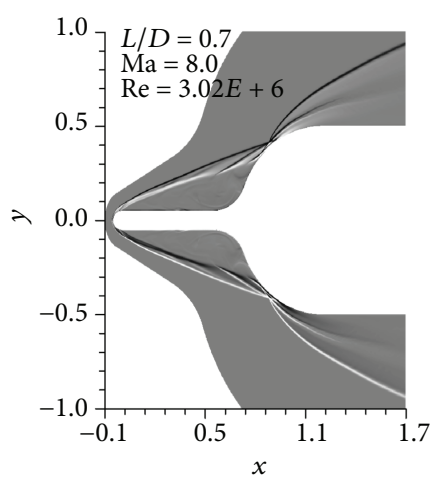

(d) $M_{\infty}=8.0$

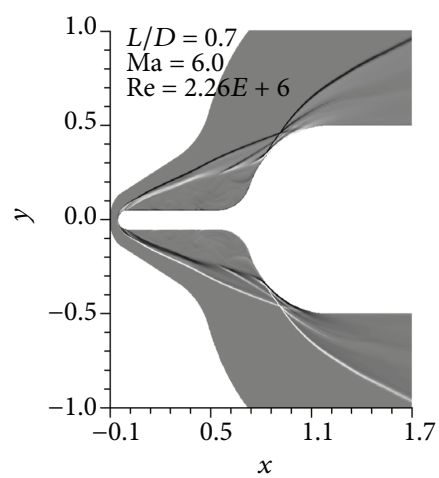

(b) $M_{\infty}=6.0$

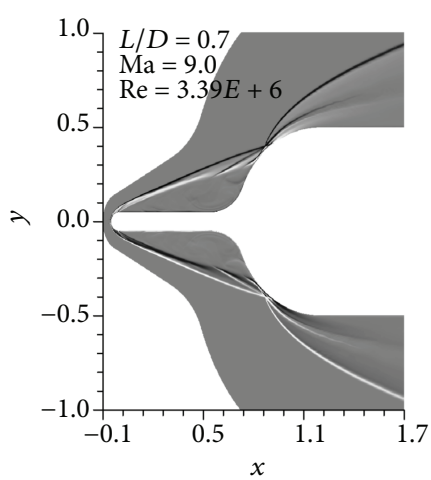

(e) $M_{\infty}=9.0$

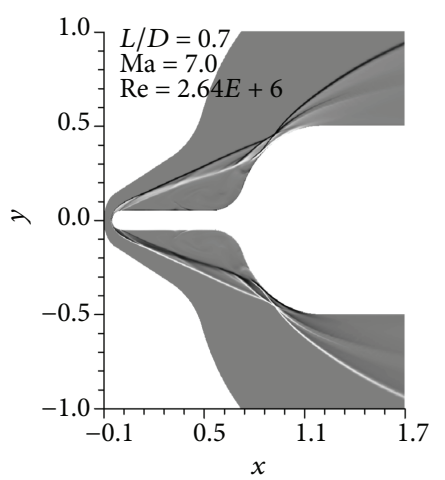

(c) $M_{\infty}=7.0$

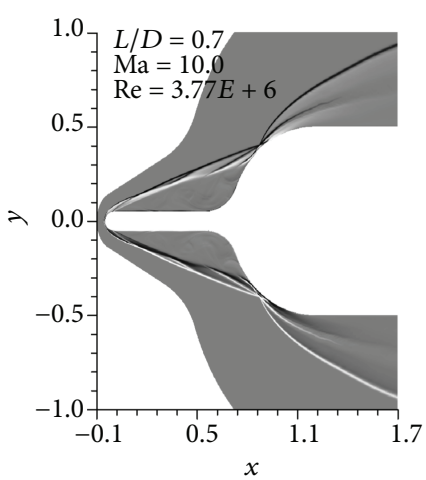

(f) $M_{\infty}=10.0$

FIGURE 10: Flow field density gradient in $y$ direction of spiked blunt bodies with the sideward sonic jet. Spike length $L / D=0.6$. 


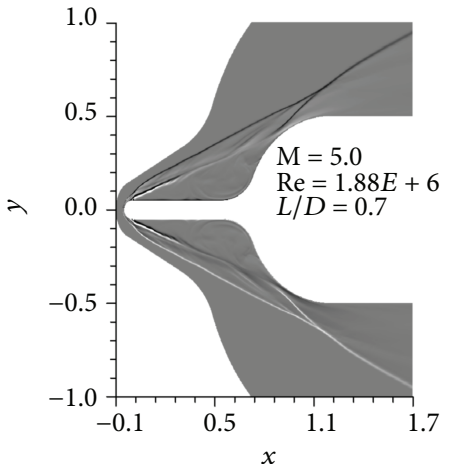

(a) $M_{\infty}=5.0$

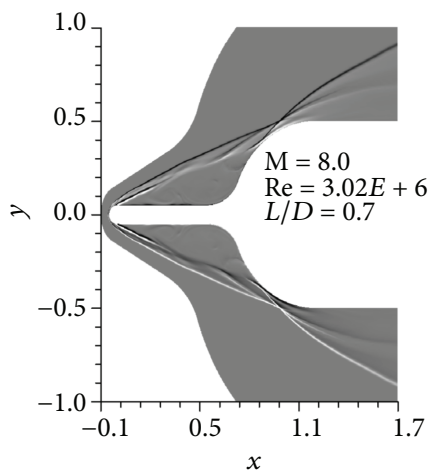

(d) $M_{\infty}=8.0$

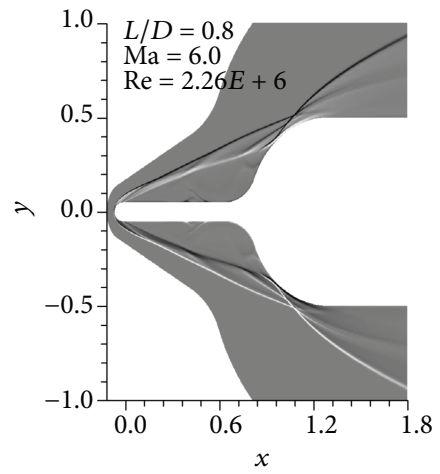

(b) $M_{\infty}=6.0$

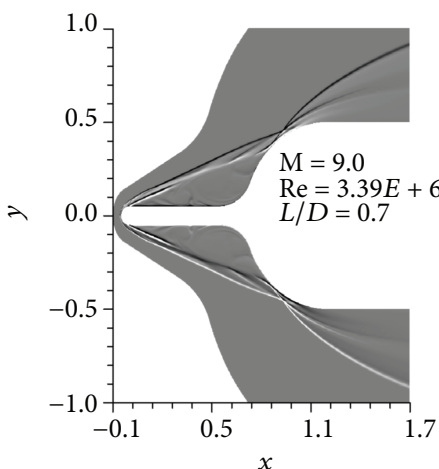

(e) $M_{\infty}=9.0$

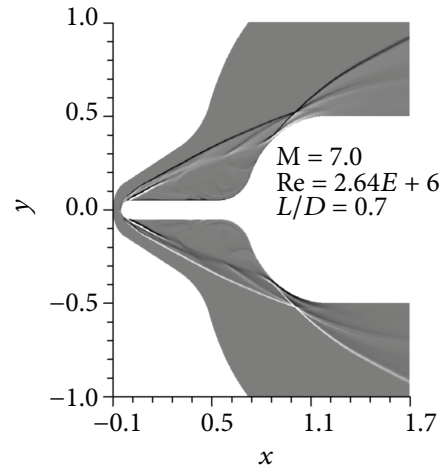

(c) $M_{\infty}=7.0$

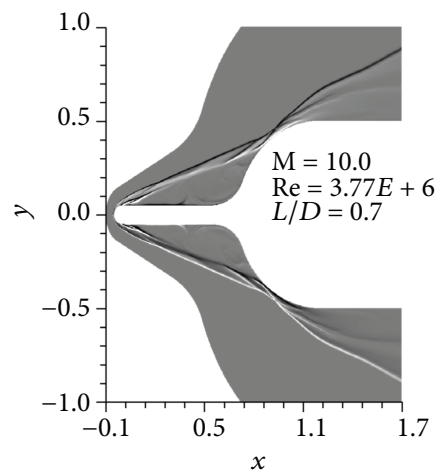

(f) $M_{\infty}=10.0$

FIGURE 11: Flow field density gradient in $y$ direction of spiked blunt bodies with the sideward sonic jet. Spike length $L / D=0.7$.

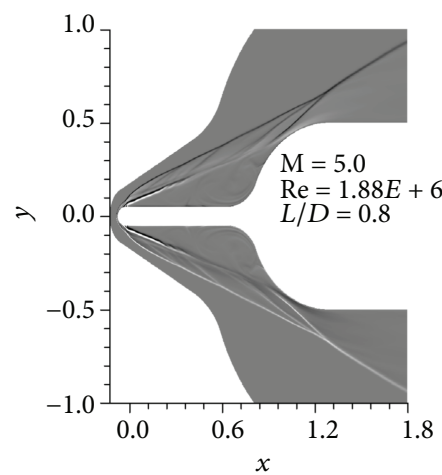

(a) $M_{\infty}=5.0$

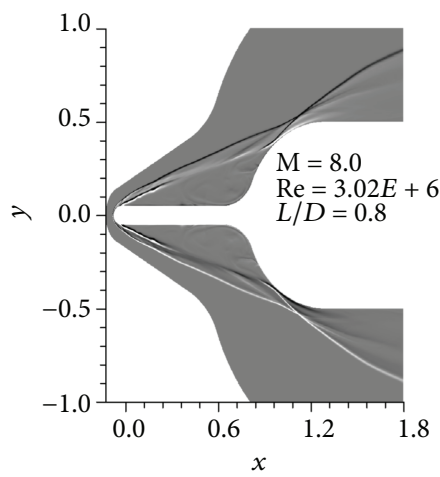

(d) $M_{\infty}=8.0$

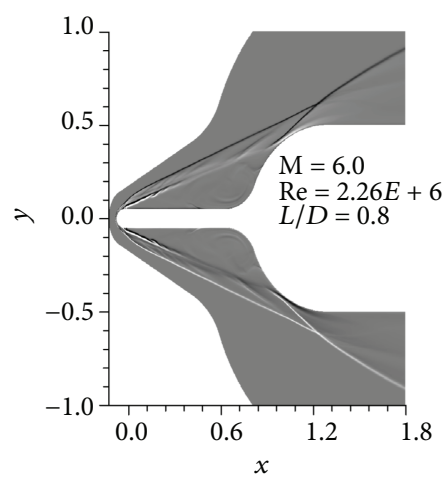

(b) $M_{\infty}=6.0$

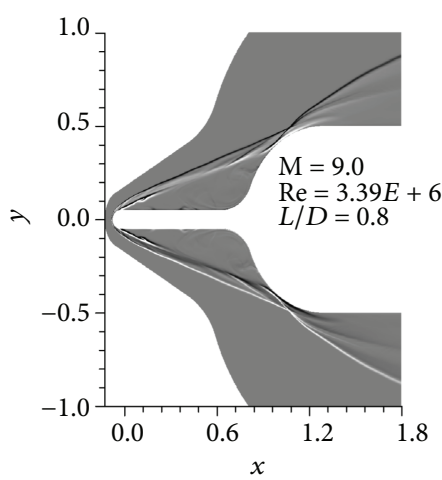

(e) $M_{\infty}=9.0$

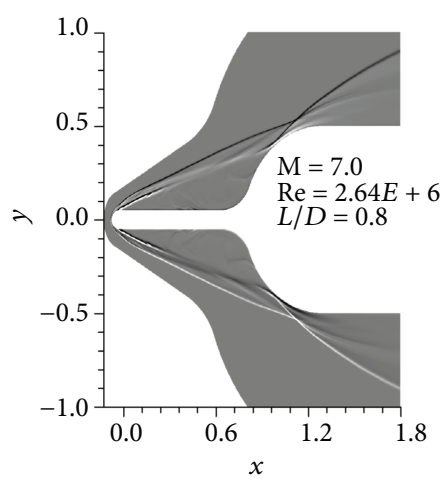

(c) $M_{\infty}=7.0$

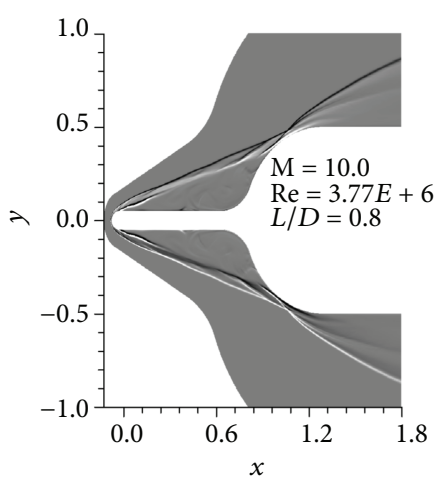

(f) $M_{\infty}=10.0$

FIGURE 12: Flow field density gradient in $y$ direction of spiked blunt bodies with the sideward sonic jet. Spike length $L / D=0.8$. 


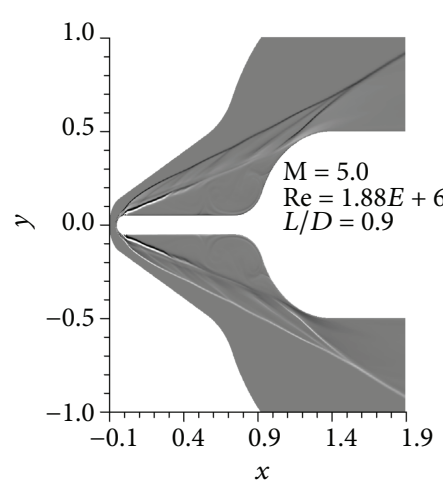

(a) $M_{\infty}=5.0$

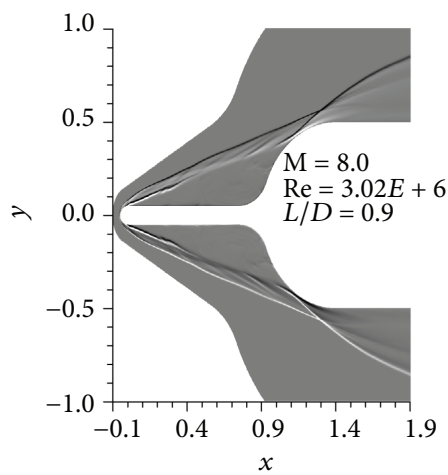

(d) $M_{\infty}=8.0$

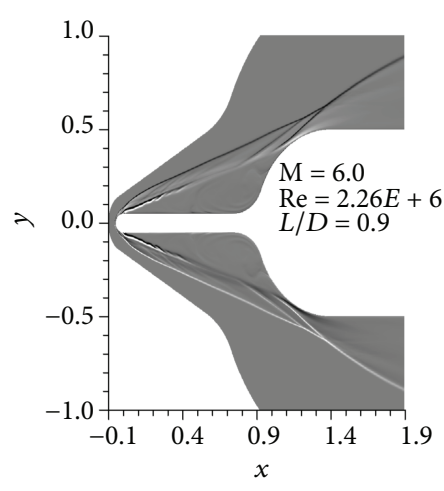

(b) $M_{\infty}=6.0$

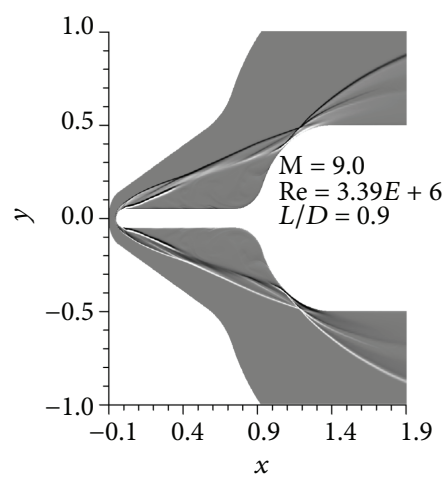

(e) $M_{\infty}=9.0$

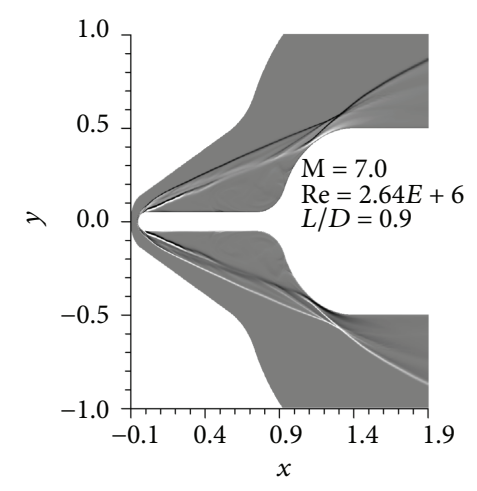

(c) $M_{\infty}=7.0$

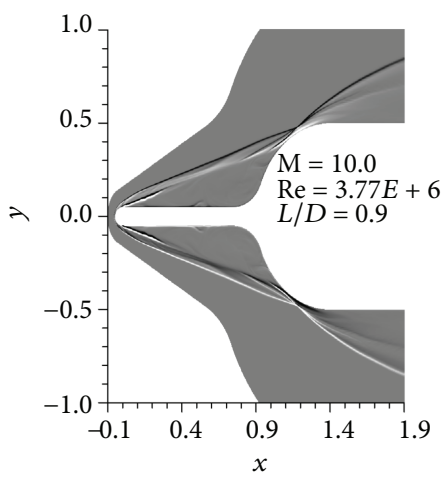

(f) $M_{\infty}=10.0$

FIGURE 13: Flow field density gradient in $y$ direction of spiked blunt bodies with the sideward sonic jet. Spike length $L / D=0.9$.

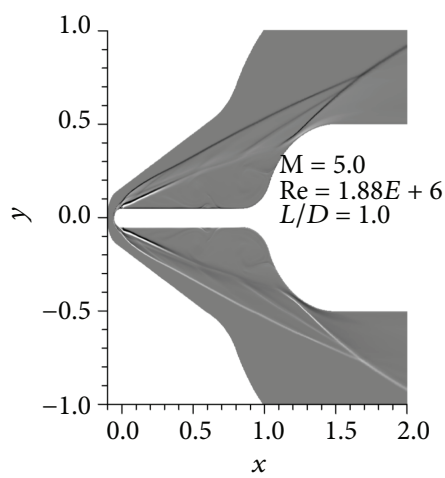

(a) $M_{\infty}=5.0$

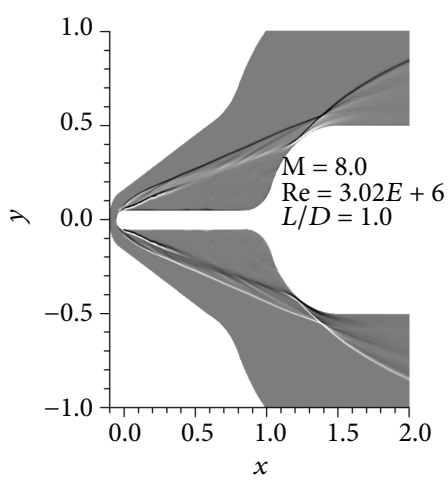

(d) $M_{\infty}=8.0$

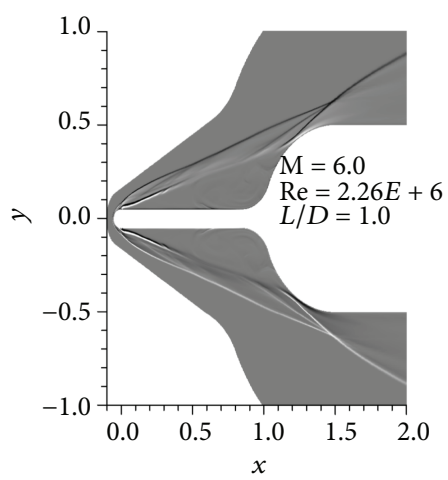

(b) $M_{\infty}=6.0$

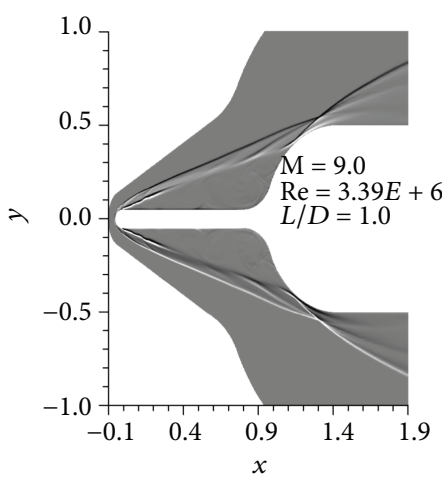

(e) $M_{\infty}=9.0$

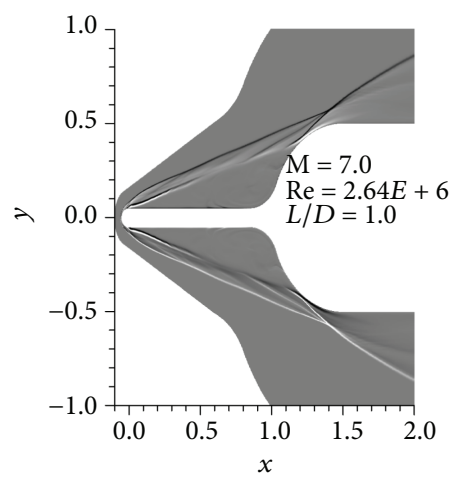

(c) $M_{\infty}=7.0$

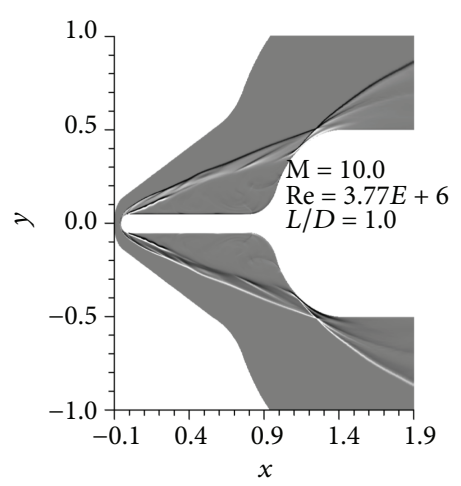

(f) $M_{\infty}=10.0$

FIGURE 14: Flow field density gradient in $y$ direction of spiked blunt bodies with the sideward sonic jet. Spike length $L / D=1.0$. 


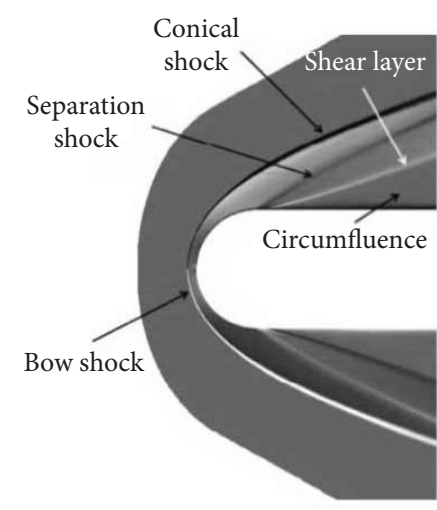

(a)

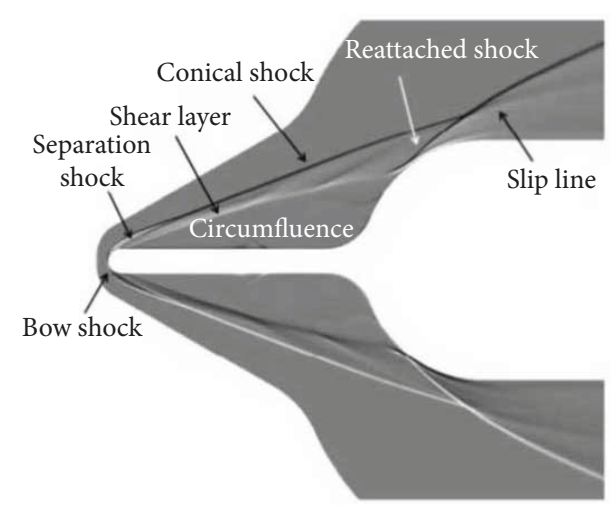

(b)

FIGURE 15: Density gradient in the $y$ direction shows typical flow field wave structure around the pure spiked blunt body $(\mathrm{Ma}=6.0, L / D=1.0)$ : (a) flow around the spike nose; (b) flow around the whole body.

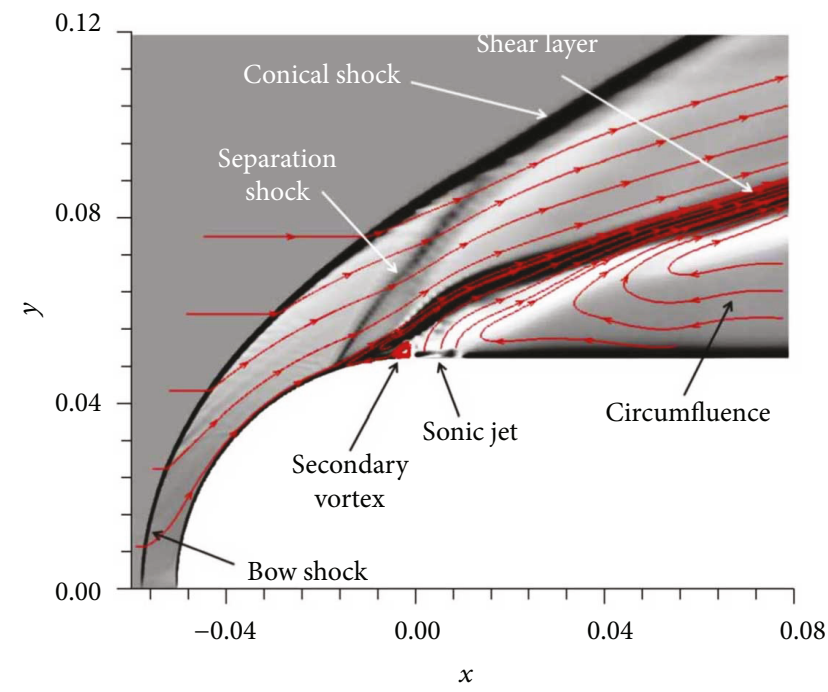

(a)

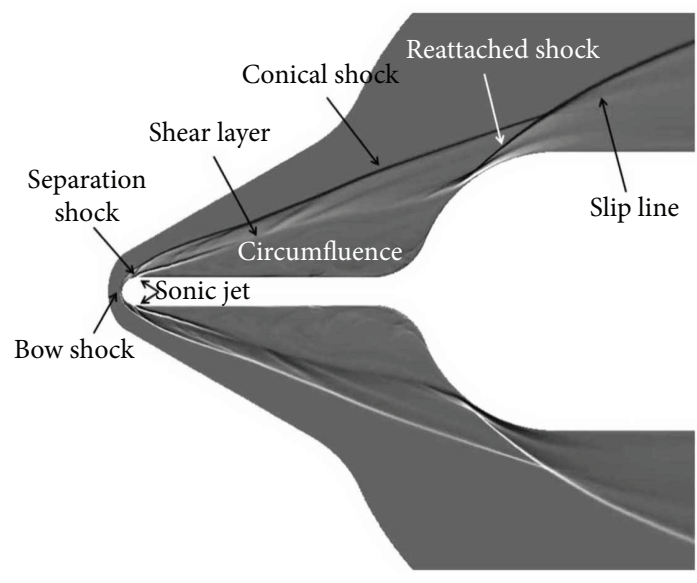

(b)

FIGURE 16: Density gradient in the $y$ direction shows typical flow field wave structure around the spiked blunt body $(\mathrm{Ma}=6.0, L / D=1.0)$ with sideward sonic jet near the spike nose: (a) flow around the spike nose; (b) flow around the whole body.

velocity was lower than that after the conical shock, generating a shear layer. The shear flow reduced some of the fluid in the circumfluence, while the reattachment fed back some fluid so that the circumfluence remained in a dynamic equilibrium state. If such an equilibrium state was broken, it causes the circumfluence and shock wave structures to vibrate. The relationship between the conical shock and reattached shock might be the real mechanism for the vibration or collapse of the circumfluence, while the former investigations took the circumfluence vibration as the unsteadiness mechanism. Due to the circumfluence, a separation shock will be generated near the spike nose before the shear layer. The conical shock interacts with the reattached shock on the blunt body shoulder, forming a slip line to match the transition between flow across two shock waves, which is exactly a shear layer due to viscosity.

The reattached shock and its interaction with conical shock were pushed away from the central line when spike length increased or the Mach number was lowered, as shown in Figures 3-8. The conical shock angle increased when the sideward sonic jet was applied near the spike nose, which pushed the interaction between conical shock and reattached shock away from the central line, as shown in the 36 figures in Figures 9-14. The sonic sideward jet affected the flow structure locally by keeping the whole macrostructure similar to that around the pure spiked blunt bodies. The detailed flow structure of the spiked blunt body with sonic sideward jet is shown in Figure 16. The sideward jet is set near the spike nose with equal velocity to local sound speed. The numerical Schlieren photograph shows that the sideward jet flows in the shear layer exchanging with the flow after conical shock and circumfluence, rather than directly flowing into the circumfluence. Due to the sideward jet, the boundary layer near the spike nose was forced to rise and flow direction was forced to swerve, putting the separation shock position forward and resulting in secondary vortex structure formation 


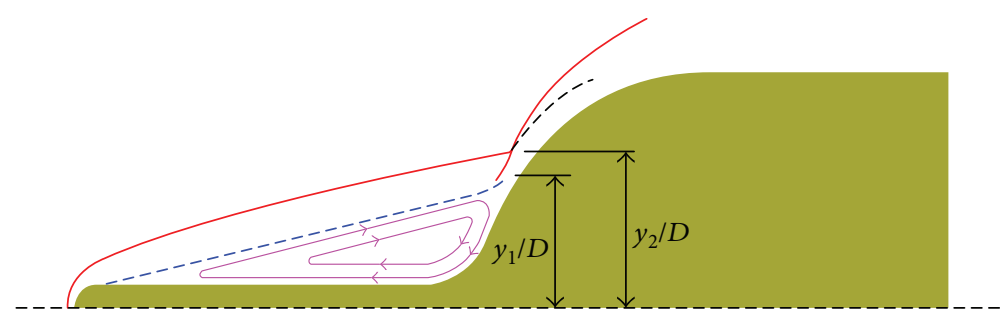

(a)

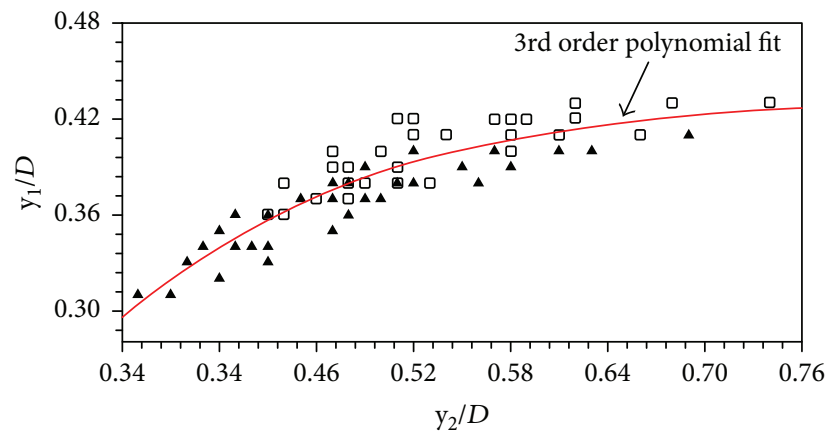

^ No jet

○ With jet

(b)

Figure 17: Two nondimensionalized parameters $y_{1} / D$ and $y_{2} / D$ for flow structure description: (a) schematic for the two parameter definitions; (b) distribution of the two parameters and a third order polynomial fit.

in the wake of the separation shock. The relative motion between the flow after separation shock and the sideward jet was transformed into one part of the shear layer due to the forward motion from the separation shock, causing the shear layer starting point to be drawn forward. The separation shock angle was increased by the sideward jet compared to the pure spike body flow field. Therefore, the angle of conical shock increased and the interaction between conical shock and reattachment shock was pushed away from the axial line. This can significantly affect the shockwave interaction on the shoulders, which is considered as a typical element of flow field reconfiguration.

3.2. Shock Wave Interaction Characteristics. Spikes reconfigured the flow field around the blunt bodies, and the interaction between shock waves was the most typical feature during the reconfiguration. The spikes set in front of the blunt bodies transformed the bow shock into a conical shock. Such a transformation must be considered as an optimization of aerodynamic characteristics, despite shock wave interactions generated on the blunt body shoulder. The main flow field configurations were similar for the pure spiked blunt bodies and spiked blunt bodies with sideward jets, including the separation shock, the conical shock, the reattached shock, the shear layer, and the circumfluence. The geometrical position of the shear layer was located near the inner tangent line connecting the spike nose and the blunt body shoulder. The flow after the conical shock was supersonic or even hypersonic, which experienced a deflection on the blunt body shoulder and generated the reattached shock. The conical shock also interacted with the reattached shock, results in the formation of a slip line, which is exactly a shear layer due to viscosity.
The main flow field characteristics can be distinguished, including the shock waves, the corresponding interactions, and the circumfluence. Two nondimensionalized parameters have been defined for the main structure description in the flow field, denoted as $y_{1} / D$ and $y_{2} / D$, as shown in Figure $17(\mathrm{a})$. The first parameter $y_{1} / D$ stands for the reattached shock position, which is equal to the distance between the reattachment position and the central line. The second parameter $y_{2} / D$ stands for the position of the interaction between conical shock and reattached shock, which is also equal to the distance to the central line. Furthermore, combined with the spike lengths, $y_{1} / D$ can also denote the shape or volume of the circumfluence generated by the flow field reconfiguration.

In this paper, 36 combinations of the incoming flow Mach numbers and the spike lengths for pure spiked bodies were taken into account so did the 36 combinations of the spiked bodies with sonic sideward jets. The data of $\left(y_{1} / D\right.$, $\left.y_{2} / D\right)$ in the 72 cases were tallied and plotted, as shown in Figure $17(\mathrm{~b})$, with $y_{2} / D$ for the horizontal coordinate axis and $y_{1} / D$ for the vertical coordinate axis. The scope of the 36 points for spiked bodies with sonic sideward jets was generally higher than that for pure spiked bodies, in both $y_{1} / D$ and $y_{2} / D$ direction of the coordinate system. It demonstrates that the sonic sideward jets led both the reattached shock and the conical shock interactions to be pushed away from the central line, affecting the aerodynamic force of the entire system. A third order polynomial can be used to fit the 72 discrete points, which generally describes a position's variation tendency of the reattached shock and its interaction. Compared with the reattached shock, the interaction between reattached shock and conical shock was more sensitive to 


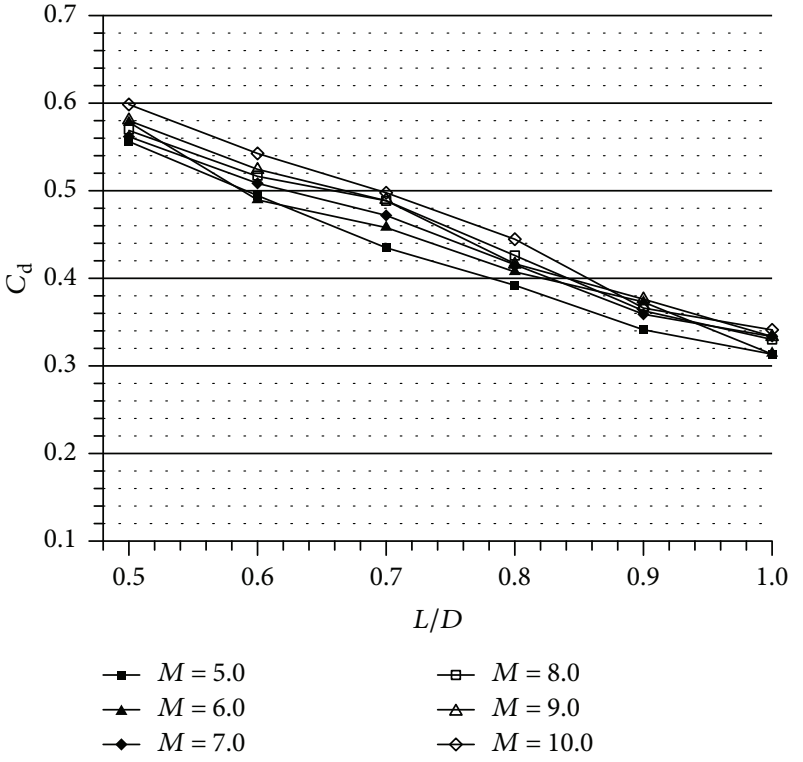

(a)

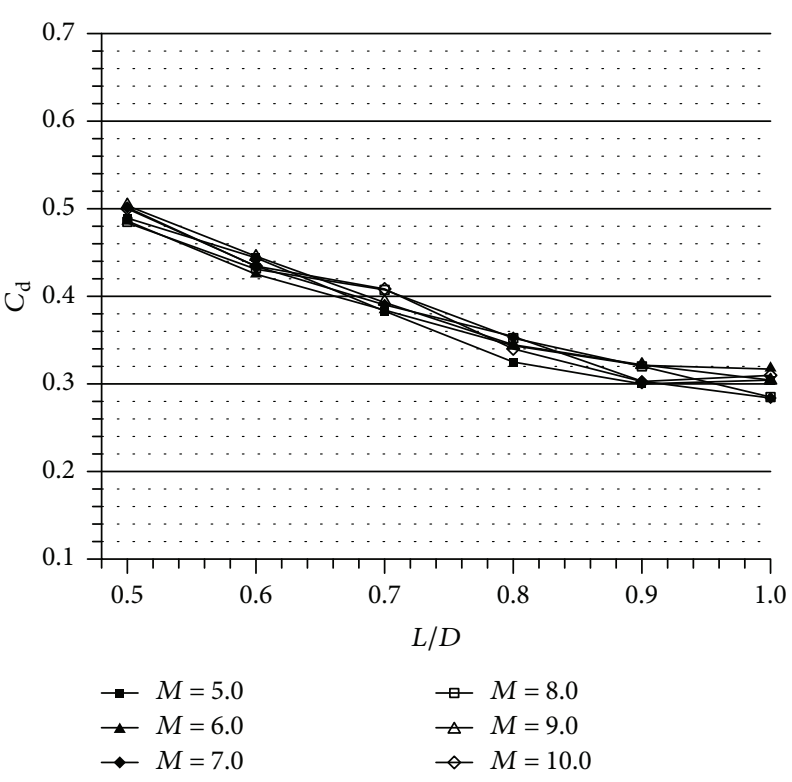

(b)

FIGURE 18: Drag coefficients vary with the spike length increased: (a) the pure spiked blunt bodies; (b) the spiked blunt bodies with sonic sideward jets.

the conditions, such as the spike lengths, the income flow Mach numbers, or the sideward jets.

The tendency of the 3rd order polynomial cure shows that there might exist a limited value of $y_{1} / D$, which maintained the reattached shock position as the spike length increased. Based on the numerical results, the limit value was about 0.43 , located at the angle about 60 degrees to the central line. Different from the reattached shock, the interaction between the conical shock and the reattached shock moved away from the central line as the spike length increased so does the slip line resulted by the interaction.

3.3. Aerodynamic Drag. The spikes reconfigure the flow field around the blunt body, changing the bow shock into three main aspects: the conical shock, the reattached shock, and the circumfluence. The flow field reconfiguration greatly reduced the shock wave drag by eliminating the strong bow shock. The reattached shock and its interaction with the conical shock will be pushed further away from the central line as the spike length increased, significantly lowering the strength and changing the aerodynamic characteristics. The drag coefficients of the pure spiked blunt bodies with 6 different spike lengths $L / D$ flying at 6 different Mach numbers are shown in Figure 18(a), where $L / D$ denotes the horizontal coordinate axis. The drag coefficient $C_{\mathrm{d}}$ decreased as $L / D$ increased from 0.5 to 1.0 , with the drag reduction of $30-40 \%$. Both the conical shock and the reattached shock are pushed to the central line as Mach number increases, which leads to an increase in drag. However, the existence of the circumfluence will prevent the conical shock and the reattached shock from being close to the central line and forming a balance. If such a balance was broken, the shocks and the circumfluence become unstable and start vibrating, during which the circumfluence seems to collapse [7]. Hence, the effect on drag by Mach number will be much less than that by the spike length in these 36 ordinary cases.

Because the wave drag plays a dominant role, the drag coefficient for a sphere flying at hypersonic speed is close to that of a sphere cylinder, which is 0.87 to 0.90 experimentally [32] and 0.87 to 0.89 in numerical results. Comparing with the pure blunt sphere cylinders, the spikes reduce wave drag as much as $35-65 \%$, which has been confirmed by other studies [15-17]. Bushnell pointed out that the effective load can be increased by $5-10 \%$ if the aerodynamic drag got discounted by as much as $1 \%$, which is likely why so many studies have been conducted regarding spiked blunt bodies [33].

The drag coefficients of the spiked blunt bodies flying at hypersonic speed with sonic sideward jets near the spike nose are shown in Figure 18(b). These include the spike lengths and Mach numbers corresponding to the 36 cases of the pure spiked blunt bodies. The effect on $C_{\mathrm{d}}$ caused by the spike length $L / D$ is similar to the cases without jets. The sonic sideward jet will push the conical shock and the reattached shock away from the central line, thus lowering the reattached shock strength and its interaction with conical shock. Therefore, the sonic sideward jet lowers spiked body drag more than the pure spiked bodies. Comparing with the 36 pure spiked blunt body cases, the sideward jets decreased the drag at a range of about 5-20\%. It was especially true for cases with short spikes. Thus, drag reduction caused by the spikes and the sonic sideward jets will be as much as $45-70 \%$ when compared with the blunt body drag. The effect on drag coefficients by the Mach number due to the sonic sideward jets is much lesser than those without the jets. Though Mach number varies, the data points 
are concentrated at the same spike length. Thus, drag reduction might increase based on the jet variety and strength, which requires further researches.

3.4. Reattached Shock and Circumfluence Criterion. The main characteristics of reconfigured flow fields have been analyzed. The reconfiguration using spikes and sonic sideward jets leads to the formation of the conical shock, the reattached shock, and the circumfluence. The roles played by each factor and the relative importance of these factors have been discussed in former sections. The aerodynamic force characteristics associated with these factors have also been analyzed. To simplify flow field description, two nondimensional geometry parameters were defined as $y_{1} / D$ and $y_{2} / D$, standing for the distance from the reattached shock and its interaction with the conical shock to the central line, respectively. Edney investigated the interaction between oblique shocks and bow shocks and classified six different types of flow fields according to interaction position and flow structure characteristics [34]. Though these shock interactions in this paper are different, flow characteristic classification can still be taken as a reference for analysis.

The interactions between the conical shock and the reattached shock in all cases, including the pure spiked bodies and the spiked bodies with sideward jets, were similar to type $\mathrm{V}$ or type VI interaction determined by different combinations of the spike length and the Mach number, as shown in Figures 3-14. The supersonic or hypersonic flow after the conical shock reattaches to the blunt body shoulder and leads to the formation of the reattached shock. The circumfluence comes from this flow reattachment due to the pressure gradient generated. The circumfluence feeds back after the conical shock, affects the flow reattachment, and lowers the strength. Therefore, a dynamical balance or couple between the reattached shock and the circumfluence was created. And both the reattached shock and the circumfluence were so stable that there was no shock interaction as strong as type IV existing in the 72 cases. However, no single parameter, including $\mathrm{Ma}$, Re, $L / D, y_{1} / D$, or $y_{2} / D$ could characterize this balance. Hence, the reattached shock and the circumfluence are preferred to be focused, based on former analysis. These two factors might be responsible for flow field reconfiguration. The balance can be quantitatively described as the distance from the reattached shock to the central line and the circumfluence volume. Therefore, a new parameter can be set up by combining these two factors, denoted as $y_{1}{ }^{2} L / D^{3}$.

The drag coefficients in the 72 cases of the pure spiked bodies and the spiked bodies with sonic sideward jets were plotted together, as shown in Figure 19. These results varied based on different horizontal coordinates. Generally, the drag coefficients decreased as the horizontal coordinate increased, remaining nearly constant when the three parameters exceeded some critical value. However, Figures 19(a) and 19(b) indicate obvious disparity among data points, suggesting that neither $y_{1} / D$ nor $y_{2} / D$ can describe the flow field reconfiguration mechanism by the spikes and the sideward jets. With $y_{1}^{2} L / D^{3}$ taken as the horizontal coordinate, the distribution of data points was significantly changed and more concentrated in the two drawn lines, as shown in

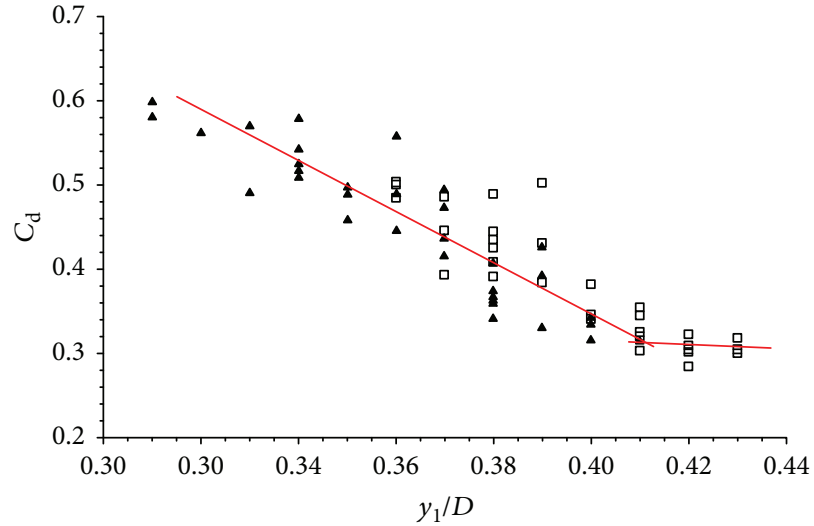

- No jet

口 With jet

(a)

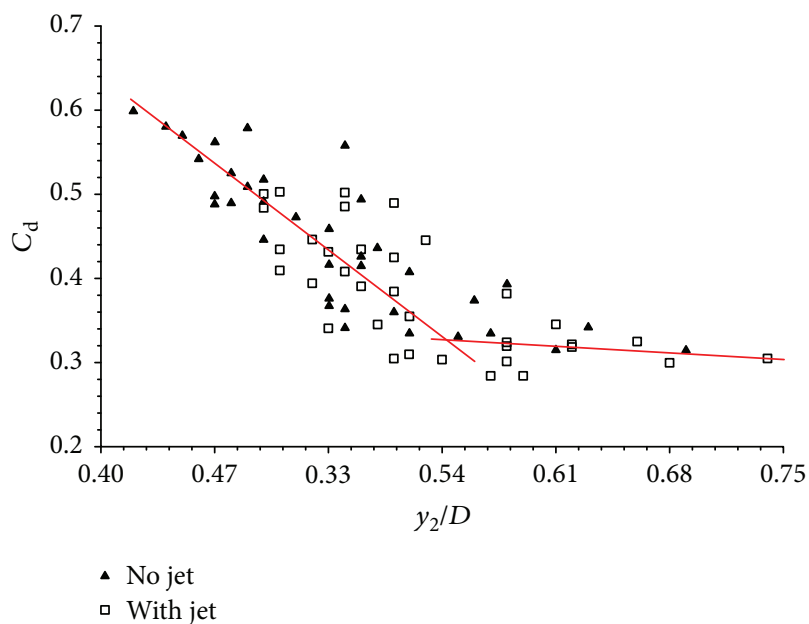

(b)

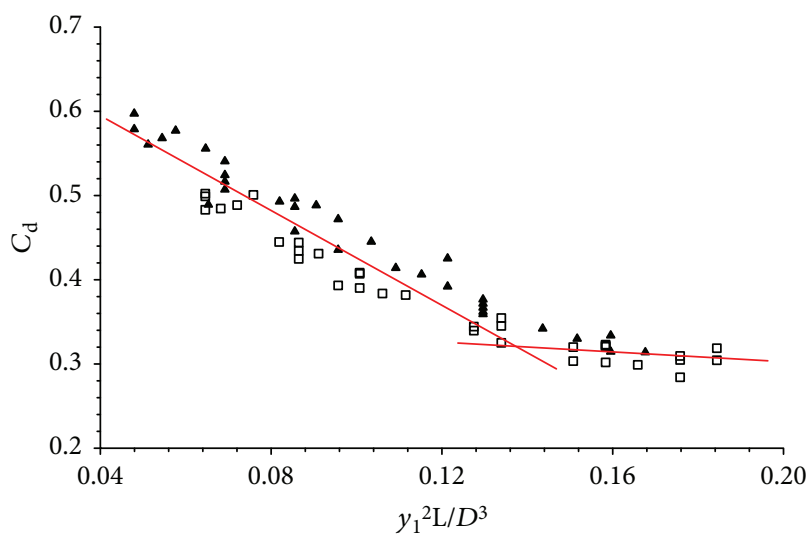

- No jet

- With jet

(c)

FIgURE 19: Drag coefficients in 72 cases of the pure spiked bodies and the spiked bodies with sonic sideward jets: (a) vary with the parameter $y_{1} / D$; (b) vary with the parameter $y_{2} / D$; (c) Vary with the combined parameter $y_{1}^{2} L / D^{3}$. 
Figure 19(c). Thus, the parameter $y_{1}^{2} L / D^{3}$ might be a simple and suitable description for the two main factors during flow field reconfiguration. In addition, drag coefficient tendency changes and becomes more gently when $y_{1}^{2} L / D^{3}$ is greater than 0.14 . The reattached shock and the circumfluence criterion for drag reduction could be used by determining the critical value of $y_{1}^{2} L / D^{3}$. Therefore, $y_{1}^{2} L / D^{3} \approx 0.14$ can be considered as a criterion for the design optimization regarding the spike length and the strength of sideward jets.

\section{Conclusions}

The 72 flow field reconfiguration cases were numerically investigated in this paper, including 36 pure spiked blunt bodies and 36 spiked bodies with sideward jets. Main flow characteristic structures and corresponding effects on aerodynamic drag have been analyzed. The spikes generated a conical shock in front of the blunt body, and the flow after conical shock reattached to the shoulder to form a reattached shock. The pressure gradient caused by the reattachment resulted in the circumfluence formation, which reversely effected the conical shock and the reattachment. Thus, a dynamical balance can be set up among the conical shock, the reattached shock, and the circumfluence, playing a dominant role in aerodynamic drag reduction. The variation tendency depends on parameter combination and might be determined in utilizing complex mechanisms, despite the force variation tendency being drawn over a single variable, such as the spike length, the position of reattached shock, and the shock/shock interaction. A criterion was primarily proposed basing on main flow structure geometric parameters $y_{1}^{2} L / D^{3}$, explicitly standing for the effect both of the reattached shock and the circumfluence. Since the connection between $y_{1} / D$ and $y_{2} / D$ can be set up utilizing a third order polynomial, the combined parameter contains the effect and strength of the conical shock and the shock interaction on the shoulder. The drag coefficient variation can be divided into two stages, and the drag reduction might be neglected when $y_{1}^{2} L / D^{3}$ is greater than 0.14 for both the pure spiked blunt bodies and the spiked bodies with sonic sideward jets.

\section{Conflicts of Interest}

The authors declare that they have no conflicts of interest.

\section{Acknowledgments}

This study was supported by the National Natural Science Foundation of China (Grant nos. 90916028, 11472281, and 11532014) and the National Key Research and Development Program (Grant no. 2016YFA0401201).

\section{References}

[1] S. M. Bogdonoff, "Preliminary investigations of spiked bodies at hypersonic speeds," Journal of the Aerospace Sciences, vol. 26, no. 2, pp. 65-74, 1959.

[2] W. E. Moeckel, "Flow separation ahead of blunt axially symmetric body at Mach number 1.76 to 2.10 ," NACA RM, article E51I25, 1951.
[3] W. E. Moeckel, "Flow separation ahead of blunt bodies at supersonic speeds," NACA TN, vol. 2418, 1951.

[4] J. R. Stadler and H. V. Nielsen, "Heat transfer from a hemisphere-cylinder equipped with flow-separation spikes," NACA TN, vol. 3287, 1954.

[5] E. C. Kennedy, "Calculation of axisymmetric isentropic spike surfaces," Journal of the Aerospace Sciences, vol. 25, no. 7, pp. 463-464, 1958.

[6] D. J. Maull, "Hypersonic flow over axially symmetric spiked bodies," Journal of Fluid Mechanics, vol. 8, no. 4, pp. 584592,1960

[7] C. J. Wood, "Hypersonic flow over spiked cones," Journal of Fluid Mechanics, vol. 12, no. 4, pp. 614-624, 1962.

[8] M. Hahn, "Pressure distribution and mass injection effects in the transitional separated flow over a spiked body at supersonic speed," Journal of Fluid Mechanics, vol. 24, no. 2, pp. 209-223, 1966.

[9] J. P. Reding, R. A. Guenther, and B. J. Richter, "Unsteady aerodynamic considerations in the design of a drag-reduction spike," Journal of Spacecraft and Rockets, vol. 14, no. 1, pp. 54-60, 1977.

[10] W. Calarese and W. L. Hankey, "Modes of shock-wave oscillations on spike-tipped bodies," AIAA Journal, vol. 23, no. 2, pp. 185-192, 1985.

[11] G. R. Hutt and A. J. Howe, "Forward facing spike effects on bodies of different cross section in supersonic flow," The Aeronautical Journal, vol. 93, no. 926, pp. 229-234, 1989.

[12] R. C. Mehta, "Numerical heat transfer study over spiked blunt bodies at Mach 6.8," Journal of Spacecraft and Rockets, vol. 37, no. 5, pp. 700-703, 2000.

[13] S. S. Milićev, M. D. Pavlović, S. Ristić, and A. Vitić, "On the influnence of spike shape at supersonic flow past blunt bodies," Facta Universitatis-Series: Mechanics, Automatic Control and Robotics, vol. 3, no. 12, pp. 371-382, 2002.

[14] A. G. Panaras and D. Drikakis, "High-speed unsteady flows around spiked-blunt bodies," Journal of Fluid Mechanics, vol. 632, pp. 69-96, 2009.

[15] V. Menezes, S. Saravanan, G. Jagadeesh, and K. P. J. Reddy, "Experimental investigations of hypersonic flow over highly blunted cones with aerospikes," AIAA Journal, vol. 41, no. 10, pp. 1955-1966, 2003.

[16] V. Menezes, S. Saravanan, and K. P. J. Reddy, "Shock tunnel study of spiked aerodynamic bodies flying at hypersonic Mach numbers," Shock Waves, vol. 12, no. 3, pp. 197-204, 2002.

[17] A. Morgenstern Jr., "Three-dimensional supersonic flow over a spike-nosed body of revolution," Journal of the Brazilian Society of Mechanical Sciences, vol. 24, no. 4, pp. 271-277, 2002.

[18] W. E. Thurman, "A flow-separation spike for hypersonic control of a hemisphere-cylinder," AIAA Journal, vol. 2, no. 1, pp. 159-161, 1964.

[19] E. Schulein, "Wave drag reduction concept for blunt bodies at high angles of attack," in 26th International Symposium on Shock Waves, Springer, Gottingen, Germany, 2007.

[20] Y. Liu and Z. Jiang, "Concept of non-ablative thermal protection system for hypersonic vehicles," AIAA Journal, vol. 51, no. 3, pp. 584-590, 2013.

[21] Y. Ma, X. Liu, and P. Ou, "Numerical investigation of hypersonic unsteady flow around a spiked blunt-body," Procedia Engineering, vol. 126, pp. 163-168, 2015. 
[22] Z. Eghlima and K. Mansour, "Drag reduction for the combination of spike and counterflow jet on blunt body at high Mach number flow," Acta Astronautica, vol. 133, pp. 103-110, 2017.

[23] A. Bibi, A. Maqsood, S. Sherbaz, and L. Dala, "Drag reduction of supersonic blunt bodies using opposing jet and nozzle geometric variations," Aerospace Science and Technology, vol. 69, pp. 244-256, 2017.

[24] W. Huang, Z. T. Zhao, L. Yan, Y. Zhou, and R. R. Zhang, "Parametric study on the drag and heat flux reduction mechanism of forward-facing cavity on a blunt body in supersonic flows," Aerospace Science and Technology, vol. 71, pp. 619626, 2017.

[25] G. L. Han, "Investigations on new concept of non-ablation and adaptive drag reduction and thermal protection system for hypersonic vehicles," Beijing: Institute of Mechanics, Chinese Academy of Sciences, 2010.

[26] K. A. Hoffmann and S. T. Chiang, Computational Fluid Dynamics for Engineers, Engineering Education SystempTM, Wichita, USA, 1993.

[27] Z. L. Jiang, K. Takayama, and Y. S. Chen, "Dispersion conditions for non-oscillatory shock capturing schemes and its applications," Computational Fluid Dynamics Journal, vol. 4, no. 2, pp. 137-150, 1995.

[28] Z. L. Jiang, "On the dispersion-controlled principles for nonoscillatory shock capturing schemes," Acta Mechanica Sinica, vol. 20, no. 1, pp. 1-15, 2004.

[29] J. L. Steger and R. F. Warming, "Flux vector splitting of the inviscid gasdynamic equations with application to finitedifference methods," Journal of Computational Physics, vol. 40, no. 2, pp. 263-293, 1981.

[30] D. X. Fu and Y. W. Ma, Computational Fluid Dynamics, Higher Education Press, Beijing, 2002.

[31] G. Ryskin and L. G. Leal, "Orthogonal mapping," Journal of Computational Physics, vol. 50, no. 1, pp. 71-100, 1983.

[32] R. N. Cox and L. F. Crabtree, Elements of Hypersonic Aerodynamics, Academic Press, New York, 1965.

[33] D. M. Bushnell, "Shock wave drag reduction," Annual Review of Fluid Mechanics, vol. 36, no. 1, pp. 81-96, 2004.

[34] B. Edney, Anomalous Heat Transfer and Pressure Distributions on Blunt Bodies at Hypersonic Speeds in the Presence of an Impinging Shock, Aeronautical Research Institute of Sweden, Report 115, Stockholm, 1968. 


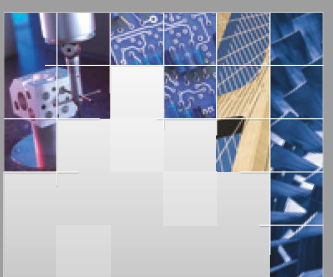

\section{Enfincering}
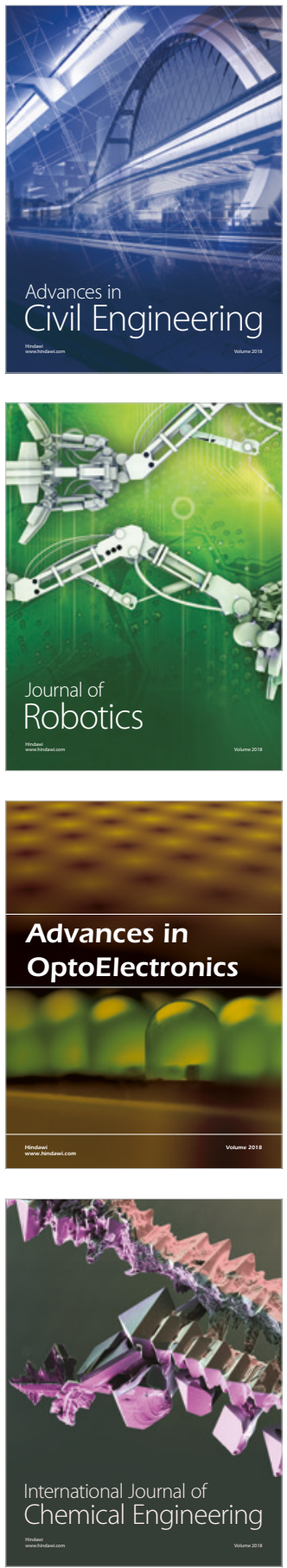

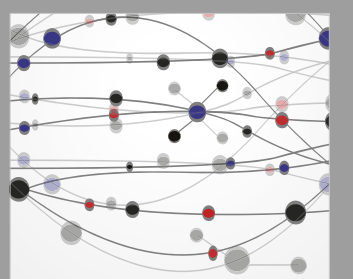

\section{Rotating \\ Machinery}

The Scientific World Journal

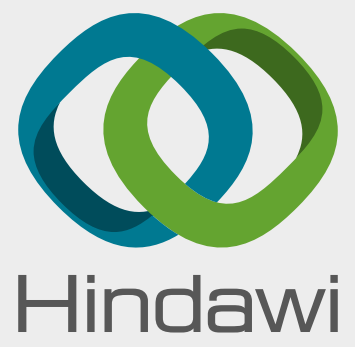

Submit your manuscripts at

www.hindawi.com
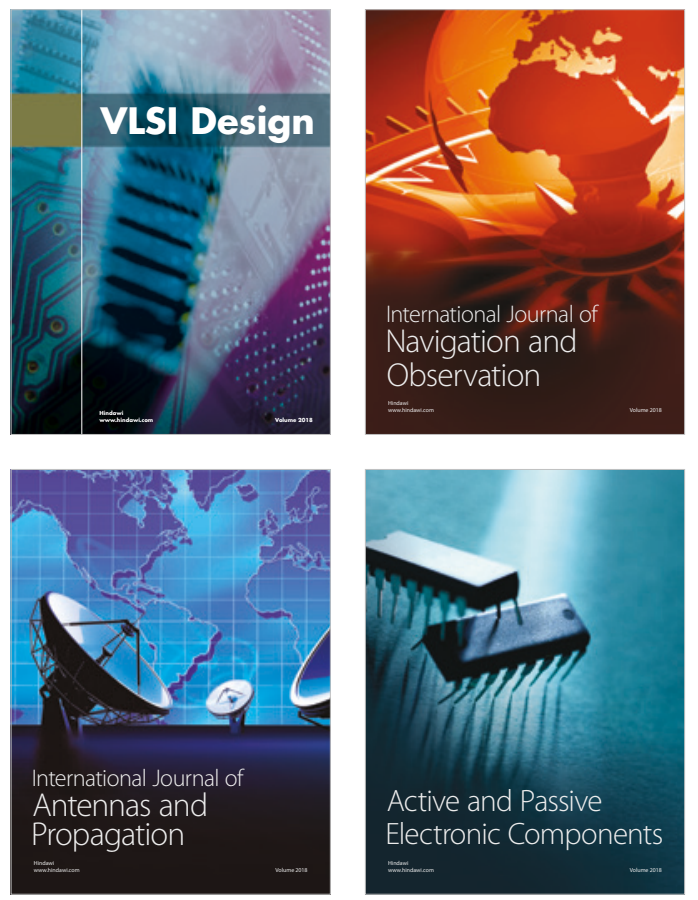
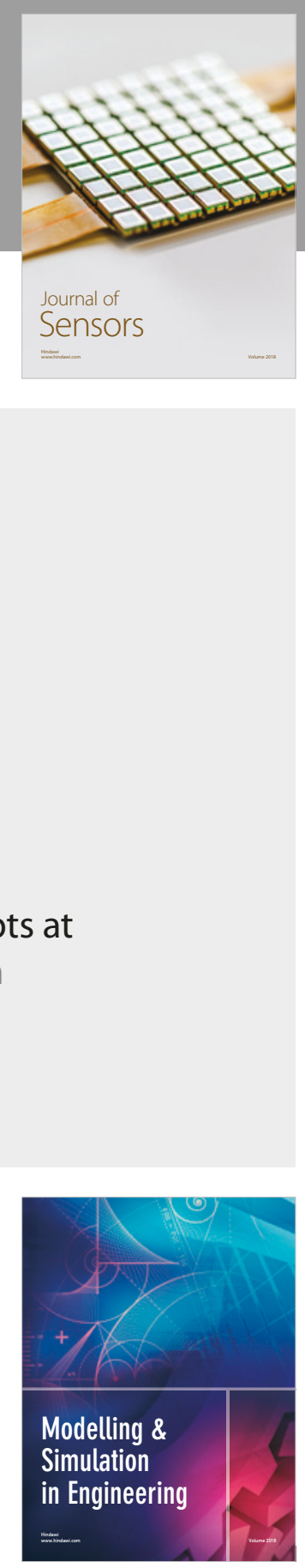

\section{Advances \\ Multimedia}
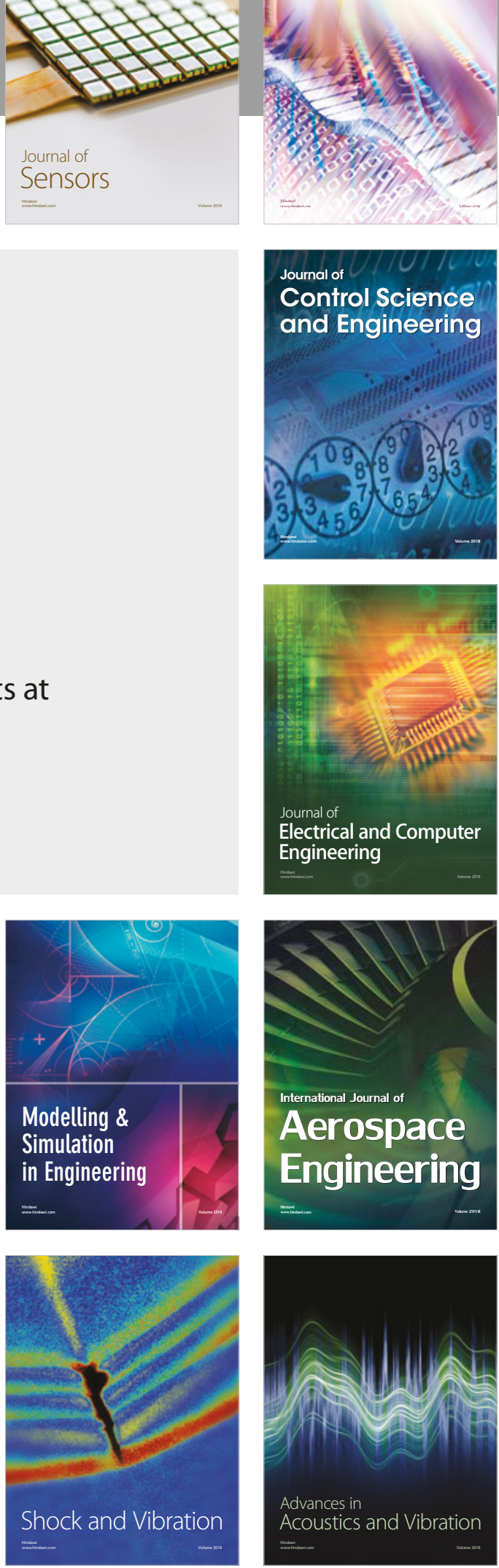